\title{
Lattice Boltzmann modeling of contact angle and its hysteresis in two-phase flow with large viscosity difference
}

\author{
Haihu Liu,* Yaping Ju, ${ }^{\dagger}$ Ningning Wang, and Guang Xi \\ School of Energy and Power Engineering, Xi'an Jiaotong University, 28 West Xianning Road, Xi'an 710049, China \\ Yonghao Zhang \\ James Weir Fluids Laboratory, Department of Mechanical 83 Aerospace Engineering, \\ University of Strathclyde, Glasgow G1 1 XJ, UK
}

(Dated: Revised manuscript received 5 August 2015; accepted 20 August 2015; published 11 September 2015)

\begin{abstract}
Contact angle hysteresis is an important physical phenomenon omnipresent in nature and various industrial processes, but its effects are not considered in many existing multiphase flow simulations due to modeling complexity. In this work, a multiphase lattice Boltzmann method (LBM) is developed to simulate the contact-line dynamics with consideration of the contact angle hysteresis for a broad range of kinematic viscosity ratios. In this method, the immiscible two-phase flow is described by a color-fluid model, in which the multiple-relaxation-time collision operator is adopted to increase numerical stability and suppress unphysical spurious currents at the contact line. The contact angle hysteresis is introduced using the strategy proposed by Ding and Spelt [Ding and Spelt, J. Fluid Mech. 599, 341 (2008)], and the geometrical wetting boundary condition is enforced to obtain the desired contact angle. This method is first validated by simulations of static contact angle and dynamic capillary intrusion process on ideal (smooth) surfaces. It is then used to simulate the dynamic behavior of a droplet on a non-ideal (inhomogeneous) surface subject to a simple shear flow. When the droplet remains pinned on the surface due to hysteresis, the steady interface shapes of the droplet quantitatively agree well with the previous numerical results. Four typical motion modes of contact points, as observed in a recent study, are qualitatively reproduced with varying advancing and receding contact angles. The viscosity ratio is found to have a notable impact on the droplet deformation, breakup and the hysteresis behavior. Finally, this method is extended to simulate the droplet breakup in a microfluidic T-junction, with one half of the wall surface ideal and the other half non-ideal. Due to the contact angle hysteresis, the droplet asymmetrically breaks up into two daughter droplets with the smaller one in the non-ideal branch channel, and the behavior of daughter droplets are significantly different in both branch channels. Also, it is found that the contact angle hysteresis is strengthened with decreasing the viscosity ratio, leading to an earlier droplet breakup and a decrease in the maximum length that the droplet can reach before the breakup. These simulation results manifest that the present multiphase LBM can be a useful substitute to Ba et al. [Ba et al., Phys. Rev. E 88, 043306 (2013)] for modeling the contact angle hysteresis, and it can be easily implemented with higher computational efficiency.
\end{abstract}

\section{INTRODUCTION}

The motion of droplets adhering to a solid surface is encountered in a wealth of natural and industrial processes such as dewdrops rolling on the leaves, inkjet printing, spray coating, enhanced oil recovery, and droplet-based microfluidics [1]. In these processes, the contact-line dynamics plays an important role in the determination of droplet behavior. Owing to its importance, the contact-line dynamics has been a long-standing subject of intensive theoretical and experimental study [1-5]. However, the contact-line dynamics is still a challenging problem that has not been fully addressed. This is partially due to the interplay of phenomena occurring over a wide range of length scales ranging from the macrosize down to the intermolecular distance.

Computational methods have emerged as promising options to describe the contact-line dynamics, and they include two major types: molecular dynamics (MD) simulations and macroscopic hydrodynamic approaches. In MD, one computes the motion of many individual molecules and their interactions [6]. The macroscopic flow properties can be obtained by an averaging process. MD provides an explicit avenue for studying the effect of solid surface properties and fluid-solid interactions [7-9]. Despite its advantages, MD calculations are extremely computationally demanding and difficult to apply at a large scale. The macroscopic hydrodynamics approaches such as volume-of-fluid $[10,11]$ and

\footnotetext{
*Electronic address: haihu.liu@mail.xjtu.edu.cn

${ }^{\dagger}$ Corresponding author. E-mail: yapingju@mail.xjtu.edu.cn
} 
level-set $[12,13]$ methods are mostly used for simulating multiphase flows with contact-line dynamics, but they require interface reconstruction or reinitialization to represent/correct the interface, which may be complex or unphysical. Moreover, an empirical slip model with slip length at the molecular scale has to be introduced in these methods to avoid stress singularities at the moving contact line [14]. Microscopically, the interface between different phases and the contact-line dynamics on the solid surface are due to interparticle interactions [15]. Thus, mesoscopic level models may be better suited to describe the complex dynamic behavior of the contact-line motion.

The lattice Boltzmann method (LBM), as a mesoscopic method, has been developed into an alternative to macroscopic hydrodynamic approaches for simulating complex fluid flow problems [16, 17]. It is a pseudo-molecular method based on particle distribution functions that performs microscopic operations with mesoscopic kinetic equations and reproduces macroscopic behavior. The LBM has several advantages over the conventional Navier-Stokes (NS) solver such as the ability to be programmed on parallel computers and the ease in dealing with complex boundaries [18]. Besides, its kinetic nature provides many of the advantages of molecular dynamics, making the LBM particularly useful for simulating multiphase, multicomponent flows. A number of multiphase, multicomponent models have been proposed in the LBM community, which can be classified into four main categories: color-fluid model [19-22], phase-field-based model [23-25], interparticle-potential model [26-28], and mean-field theory model [29]. For a comprehensive review of these models, interested readers may refer to Refs. [18, 30]. Among these models, the color-fluid model was extensively used to simulate immiscible multiphase flow problems [31-33] because of its advantages such as low spurious currents, high numerical accuracy and strict mass conservation for each fluid. In particular, it has been extended recently to model the contact-line dynamics with the contact angle hysteresis [34], which is essentially inherent to contact-line motion. In this model, the color-conserving wetting boundary condition [35] was applied to describe the dynamic evolution of the contact line, and a modified numerical algorithm originally proposed in the VOF-based method [36] was presented to account for the contact angle hysteresis. This model is effective and accurate in modeling the contact angle hysteresis, but its implementations are extremely complicated and it suffers from the difficulty of handling complex boundaries.

The contact angle hysteresis is the difference between the advancing and receding contact angles and is used to characterize surface roughness and/or surface heterogeneity. The contact angle hysteresis is important to the understanding of droplet motion and to controlling further droplet dynamical behavior on a solid surface. In this work, we present a color-fluid LBM for the simulation of contact angle and its hysteresis behavior, with an emphasis on the capability of simulating binary fluids with large viscosity difference/ratio. The immiscible two-phase flows are modeled after the work of Halliday et al. [20] except that the original BGK collision operator is replaced by its multiplerelaxation-time (MRT) counterpart in order to minimize spurious currents and increase the numerical stability of the model at large viscosity ratio [37-39]. To alleviate the complexity and difficulty associated to the previous model, the contact angle hysteresis is incorporated into the LBM by using the strategy of Ding and Spelt [40], in which the desired contact angle is enforced through the geometrical formulation proposed in Ref. [41]. The capability and accuracy of this method are tested by several typical flow cases, including simulations of static contact angle, dynamic capillary intrusion process on an ideal surface, the dynamic behavior of a droplet on a non-ideal surface subjected to a shear flow, and the asymmetric droplet breakup caused by contact angle hysteresis at a T-junction.

\section{METHODOLOGY}

\section{A. Lattice Boltzmann color-fluid model for immiscible two-phase flow}

The immiscible two-phase flow is modeled through a MRT color-fluid model, which is developed on the basis of the work by Halliday and his coworkers $[20,42,43]$. In this model, red and blue distribution functions $f_{i}^{R}$ and $f_{i}^{B}$ are introduced to represent two different fluids. The total distribution function is defined by $f_{i}=f_{i}^{R}+f_{i}^{B}$, which undergoes a collision step as

$$
f_{i}^{\dagger}(\vec{x}, t)=f_{i}(\vec{x}, t)+\Omega_{i}(\vec{x}, t)+\bar{F}_{i}
$$

where $f_{i}(\vec{x}, t)$ is the total distribution function in the $i$-th velocity direction at the position $\vec{x}$ and time $t, f_{i}^{\dagger}$ is the post-collision distribution function, $\Omega_{i}$ is the collision operator, and $\bar{F}_{i}$ is the forcing term. Instead of using the BGK approximation as reported in Refs. [20, 34,44], the MRT model is adopted for the collision operator, which is given by $[45,46]$

$$
\Omega_{i}(\vec{x}, t)=-\left(\mathbf{M}^{-1} \mathbf{S M}\right)_{i j}\left[f_{j}(\vec{x}, t)-f_{j}^{e q}(\vec{x}, t)\right]
$$

where $f_{i}^{e q}$ is the equilibrium distribution functions of $f_{i}, \mathbf{M}$ is a transformation matrix, and $\mathbf{S}$ is a diagonal relaxation matrix. The equilibrium distribution function is obtained by a second order Taylor expansion of Maxwell-Boltzmann 
distribution with respect to the local fluid velocity $\vec{u}$ :

$$
f_{i}^{e q}=\rho w_{i}\left[1+\frac{\vec{e}_{i} \cdot \vec{u}}{c_{s}^{2}}+\frac{\left(\vec{e}_{i} \cdot \vec{u}\right)^{2}}{2 c_{s}^{4}}-\frac{\vec{u}^{2}}{2 c_{s}^{2}}\right],
$$

where $\rho=\rho_{R}+\rho_{B}$ is the total density with the subscripts ' $\mathrm{R}$ ' and ' $\mathrm{B}$ ' referring to the red and blue fluids respectively; $c_{s}$ is the speed of sound; $\vec{e}_{i}$ is the lattice velocity in the $i$-th direction; and $w_{i}$ is the weight factor. For the twodimensional 9-velocity (D2Q9) model [47], $\vec{e}_{i}$ is defined as $\vec{e}_{0}=(0,0), \vec{e}_{1,3}=( \pm c, 0), \vec{e}_{2,4}=(0, \pm c), \vec{e}_{5,7}=( \pm c, \pm c)$, and $\vec{e}_{6,8}=(\mp c, \pm c)$, where $c=\delta_{x} / \delta_{t}=\sqrt{3} c_{s}$ with $\delta_{x}$ being the lattice spacing and $\delta_{t}$ being the time step; $w_{i}$ is given by $w_{0}=4 / 9, w_{1-4}=1 / 9$ and $w_{5-8}=1 / 36$.

The forcing term contributes to the mixed interfacial regions and generates an interfacial tension. In the MRT framework, the forcing term reads as [48]

$$
\overline{\mathbf{F}}=\mathbf{M}^{-1}\left(\mathbf{I}-\frac{1}{2} \mathbf{S}\right) \mathbf{M} \tilde{\mathbf{F}},
$$

where $\mathbf{I}$ is a $9 \times 9$ unit matrix, $\overline{\mathbf{F}}=\left[\bar{F}_{0}, \bar{F}_{1}, \bar{F}_{2}, \ldots, \bar{F}_{8}\right]^{T}$, and $\tilde{\mathbf{F}}=\left[\tilde{F}_{0}, \tilde{F}_{1}, \tilde{F}_{2}, \ldots, \tilde{F}_{8}\right]^{T}$ is given by

$$
\tilde{F}_{i}=w_{i}\left[\frac{\vec{e}_{i}-\vec{u}}{c_{s}^{2}}+\frac{\left(\vec{e}_{i} \cdot \vec{u}\right) \vec{e}_{i}}{c_{s}^{4}}\right] \cdot \vec{F} \delta_{t} .
$$

In the above equation, $\vec{F}$ is the body force, which is responsible for producing the local stress jump across the interface. Based on the continuum surface force (CSF) model of Brackbill et. al [49], the body force can be expressed as [42]

$$
\vec{F}(\vec{x}, t)=-\frac{1}{2} \sigma \kappa \nabla \rho^{N}
$$

where $\sigma$ is an interfacial tension parameter, $\rho^{N}$ is the phase field defined by

$$
\rho^{N}(\vec{x}, t)=\frac{\rho_{R}(\vec{x}, t)-\rho_{B}(\vec{x}, t)}{\rho_{R}(\vec{x}, t)+\rho_{B}(\vec{x}, t)}, \quad-1 \leq \rho^{N} \leq 1,
$$

and $\kappa$ is the local interface curvature, which is calculated by

$$
\kappa=-[(\mathbf{I}-\vec{n} \otimes \vec{n}) \cdot \nabla] \cdot \vec{n}=-\nabla \cdot \vec{n},
$$

where $\vec{n}$ is the unit normal vector defined by $\vec{n}=-\frac{\nabla \rho^{N}}{\left|\nabla \rho^{N}\right|}$.

According to the previous studies, e.g. Refs. [50, 51], the local fluid velocity should be defined to incorporate the spatially varying body force, i.e.,

$$
\rho \vec{u}(\vec{x}, t)=\sum_{i} f_{i}(\vec{x}, t) \vec{e}_{i}+\frac{1}{2} \vec{F}(\vec{x}, t) \delta_{t} .
$$

The transformation matrix $\mathbf{M}$ is constructed by the Gram-Schmidt orthogonalization procedure from the discrete velocity set, and is given explicitly by [45]

$$
\mathbf{M} \equiv\left(\begin{array}{c}
\langle\rho| \\
\langle e| \\
\langle\varepsilon| \\
\left\langle j_{x}\right| \\
\left\langle q_{x}\right| \\
\left\langle j_{y}\right| \\
\left\langle q_{y}\right| \\
\left\langle p_{x x}\right| \\
\left\langle p_{x y}\right|
\end{array}\right)=\left(\begin{array}{ccccccccc}
1 & 1 & 1 & 1 & 1 & 1 & 1 & 1 & 1 \\
-4 & -1 & -1 & -1 & -1 & 2 & 2 & 2 & 2 \\
4 & -2 & -2 & -2 & -2 & 1 & 1 & 1 & 1 \\
0 & 1 & 0 & -1 & 0 & 1 & -1 & -1 & 1 \\
0 & -2 & 0 & 2 & 0 & 1 & -1 & -1 & 1 \\
0 & 0 & 1 & 0 & -1 & 1 & 1 & -1 & -1 \\
0 & 0 & -2 & 0 & 2 & 1 & 1 & -1 & -1 \\
0 & 1 & -1 & 1 & -1 & 0 & 0 & 0 & 0 \\
0 & 0 & 0 & 0 & 0 & 1 & -1 & 1 & -1
\end{array}\right),
$$

where the Dirac notation of bra $\langle\cdot|$ symbolizes the 9-dimensional row vector. Note that the row vectors of $\mathbf{M}$ are orthogonal to each other but they are not normalized. With the transformation matrix $\mathbf{M}$, the particle distribution function $f_{i}$ can be projected onto the moment space through $m_{i}=\mathbf{M}_{i j} f_{j}$, and the resulting nine moments are

$$
\mathbf{m} \equiv\left(m_{0}, m_{1}, \ldots, m_{8}\right)^{\mathrm{T}}=\left(\rho, e, \varepsilon, j_{x}, q_{x}, j_{y}, q_{y}, p_{x x}, p_{x y}\right)^{\mathrm{T}}
$$


where $e$ and $\varepsilon$ are related to the total energy and the energy square, $j_{x}$ and $j_{y}$ are the $x$ - and $y$-components of the momentum, i.e. $j_{x}=\rho u_{x}$ and $j_{y}=\rho u_{y}, q_{x}$ and $q_{y}$ are the $x$ - and $y$-components of the energy flux, and $p_{x x}$ and $p_{x y}$ are related to the symmetric and traceless components of the stress tensor, respectively. The diagonal relaxation matrix $\mathbf{S}$ is defined as

$$
\mathbf{S}=\operatorname{diag}\left[s_{0}, s_{1}, s_{2}, s_{3}, s_{4}, s_{5}, s_{6}, s_{7}, s_{8}\right]
$$

where the elements $s_{i}$ are the relaxation rates associated with each $f_{i}$. The parameters $s_{0}, s_{3}$ and $s_{5}$ correspond to the conserved moments (i.e., $\rho, j_{x}$ and $j_{y}$ ) and have no effect on the derivation of the NS equations [52]. For simplicity, we choose $s_{0}=s_{3}=s_{5}=1$. $s_{1}$ determines the bulk viscosity $\zeta$ through

$$
\zeta=\left(\frac{1}{s_{1}}-\frac{1}{2}\right) c_{s}^{2} \delta_{t},
$$

and it is considered as an adjustable parameter since the binary fluids are incompressible. $s_{7}$ and $s_{8}$ are related to the kinematic viscosity $\nu$ by

$$
s_{7}=s_{8}=\frac{1}{\tau}, \quad \text { and } \nu=\left(\tau-\frac{1}{2}\right) c_{s}^{2} \delta_{t}
$$

Besides, symmetry requires that $s_{4}=s_{6}$. Consequently, three independent parameters $s_{1}, s_{2}$ and $s_{4}\left(=s_{6}\right)$ can be freely adjusted to enhance the stability of MRT model [46, 48, 53, 54]. Following the guidelines and suggestions in Ref. [45], we choose these free parameters as $s_{1}=1.63, s_{2}=1.14$, and $s_{4}=s_{6}=1.92$ in this study. It was also demonstrated that such a choice can effectively suppress unphysical spurious currents in the vicinity of the contact line, resulting in an increased numerical accuracy in simulating contact angles [39].

Using the Chapman-Enskog multiscale analysis, Eq. (1) can be reduced to the NS equations in the low frequency, long wavelength limit with Eqs. (2)-(5). The resulting equations are

$$
\begin{gathered}
\partial_{t} \rho+\nabla \cdot(\rho \mathbf{u})=0, \\
\partial_{t}(\rho \vec{u})+\nabla \cdot(\rho \vec{u} \vec{u})=-\nabla p+\nabla \cdot\left[\mu\left(\nabla \vec{u}+\nabla \vec{u}^{T}\right)\right]+\vec{F},
\end{gathered}
$$

where $p=\rho c_{s}^{2}$ is the pressure, and $\mu=\rho \nu$ is the dynamic viscosity of the fluid mixture. In this study, the pure red and blue fluids are assumed to have equal densities, i.e., $\tilde{\rho}_{R}=\tilde{\rho}_{B}$. To allow for unequal viscosities of the two fluids, we determine the viscosity of the fluid mixture by a harmonic mean $[33,55]$ :

$$
\frac{1}{\mu\left(\rho^{N}\right)}=\frac{1+\rho^{N}}{2 \mu_{R}}+\frac{1-\rho^{N}}{2 \mu_{B}},
$$

where $\mu_{k}(k=R$ or $B)$ is the dynamic viscosity of fluid $k$, which is related to the kinematic viscosity $\nu_{k}$ by $\mu_{k}=\tilde{\rho}_{k} \nu_{k}$. In the present diffuse-interface model, the choice of Eq. (17) can ensure a constant viscous stress across the interface, which can be explained as follows. Consider a horizontal interface in the $x-y$ plane that separates the red and blue fluids on both sides. The viscous stress reads as $\mu \partial_{y} u_{x}$ [55], which should be a constant across the interface based on a force balance. Note that the velocity gradient $\partial_{y} u_{x}$ is variable across the interface when both fluids have unequal viscosities. We define $\left(\partial_{y} u_{x}\right)_{R}$ and $\left(\partial_{y} u_{x}\right)_{B}$ as the velocity gradients on the red and blue fluid sides, respectively. Besides, we simply take the velocity gradient at the interface as $\frac{1+\rho^{N}}{2}\left(\partial_{y} u_{x}\right)_{R}+\frac{1-\rho^{N}}{2}\left(\partial_{y} u_{x}\right)_{B}$ by assuming that the velocity gradient varies linearly with the local phase field. A constant viscous stress across the interface requires $\mu\left[\frac{1+\rho^{N}}{2}\left(\partial_{y} u_{x}\right)_{R}+\frac{1-\rho^{N}}{2}\left(\partial_{y} u_{x}\right)_{B}\right]=\mu_{R}\left(\partial_{y} u_{x}\right)_{R}=\mu_{B}\left(\partial_{y} u_{x}\right)_{B}$, which can easily lead to Eq. (17). It is worth noting that the harmonic mean was also used for the interface viscosity to ensure the continuity of viscous stress in the sharp-interface models $[56,57]$.

The partial derivatives required for the curvature and normal vector calculations are obtained using the 9-point compact finite-difference stencil. For example, for a variable $\psi$, its partial derivatives can be evaluated by [22]

$$
\frac{\partial \psi(\vec{x})}{\partial x_{\alpha}}=\frac{1}{c_{s}^{2}} \sum_{i} w_{i} \psi\left(\vec{x}+\vec{e}_{i} \delta_{t}\right) e_{i \alpha} .
$$

Although the forcing term generates an interfacial tension, it does not guarantee the immiscibility of both fluids. To promote phase segregation and maintain the interface, we use the recoloring algorithm proposed by Latva-Kokko 
and Rothman [58], which helps further reduce spurious currents at the interface and overcome the lattice pinning problem arising in the original recoloring algorithm of Gunstensen et al. [19]. Following Latva-Kokko and Rothman, the recolored distribution functions of the red and blue fluids are [58]

$$
\begin{aligned}
f_{i}^{R}(\vec{x}, t) & =\frac{\rho_{R}}{\rho} f_{i}^{\dagger}(\vec{x}, t)+\beta \frac{\rho_{R} \rho_{B}}{\rho} w_{i} \cos \left(\varphi_{i}\right)\left|\mathbf{e}_{i}\right|, \\
f_{i}^{B}(\vec{x}, t) & =\frac{\rho_{B}}{\rho} f_{i}^{\dagger}(\vec{x}, t)-\beta \frac{\rho_{R} \rho_{B}}{\rho} w_{i} \cos \left(\varphi_{i}\right)\left|\mathbf{e}_{i}\right|,
\end{aligned}
$$

where $\beta$ is the segregation parameter and is set to be 0.7 for numerical stability and model accuracy $[22,31,43] ; \varphi_{i}$ is the angle between the phase field gradient and the lattice vector $\vec{e}_{i}$, which is defined by

$$
\cos \left(\varphi_{i}\right)=\frac{\vec{e}_{i} \cdot \nabla \rho^{N}}{\left|\vec{e}_{i}\right|\left|\nabla \rho^{N}\right|}
$$

After the recoloring step, the distribution functions propagate to the neighbouring lattice nodes, known as propagation or streaming step:

$$
f_{i}^{k}\left(\vec{x}+\vec{e}_{i} \delta_{t}, t+\delta_{t}\right)=f_{i}^{k}(\vec{x}, t), \quad k=R \text { or } B
$$

with the post-propagation distribution functions being used to calculate the densities of both fluids by $\rho^{k}=\sum_{i} f_{i}^{k}$.

\section{B. Wetting boundary condition}

As for the color-fluid LBM, we have recently developed a wetting boundary condition to model fluid-surface interactions [34]. Our wetting boundary condition consists of three parts: (1) a contact angle model that enforces the specified contact angle at the solid wall, (2) a color-conserving boundary closure scheme that solves the post-collision distribution functions by enforcing no-slip boundary condition and mass conservation for each fluid, and (3) a variant of recoloring algorithm that is designed to maintain the reasonable interface at the solid wall. It has been numerically demonstrated to be capable of modeling the contact-line dynamics with high accuracy and low spurious currents. However, its implementation is relatively complex and it is hard to be extended for complex boundaries. In this work, the wetting boundary condition is implemented in a much simpler manner, as described previously by Huang et al. [59] for phase-field-based LBM simulations.

No-slip boundary condition is applied at the solid wall by using the halfway bounce-back scheme [60], where the wall is located halfway between the fluid node and solid node. Following our previous work [34, 61], the contact angle $\theta$ is enforced at the wall through the geometrical formulation proposed by Ding and Spelt [41], i.e.,

$$
\vec{n}_{w} \cdot \nabla \rho^{N}=-\Theta_{w}\left|\vec{t}_{w} \cdot \nabla \rho^{N}\right|
$$

with

$$
\Theta_{w}=\tan \left(\frac{\pi}{2}-\theta\right)
$$

where $\vec{n}_{w}$ is the unit normal vector to wall pointing towards the fluids, and $\vec{t}_{w}$ is the unit vector tangential to wall. The enforcement of Eq. (22) is realized through the external solid nodes (also termed as ghost nodes), which are a half lattice spacing away from the wall. Without losing generality, we consider the case of bottom wall, whose neighboring solid and fluid nodes are located at $y=0$ and $y=1$, respectively. Upon discretization of Eq. (22), one has

$$
\rho_{x, 0}^{N}=\rho_{x, 1}^{N}+\Theta_{w}\left|\vec{t}_{w} \cdot \nabla \rho^{N}\right| \delta_{x}
$$

where the tangential component of $\nabla \rho^{N}$ on the wall is determined by the following extrapolation scheme [59]:

$$
\vec{t}_{w} \cdot \nabla \rho^{N}=\left.1.5 \partial_{x} \rho^{N}\right|_{x, 1}-\left.0.5 \partial_{x} \rho^{N}\right|_{x, 2} .
$$

In Eq. (25), the derivatives on the right-hand side can be easily evaluated by the second-order central difference approximation. Once the value of the phase field $\rho_{x, 0}^{N}$ is obtained, the partial derivatives of $\rho^{N}$, appearing in Eqs. (6), at all the fluid nodes can be calculated uniformly by Eq. (18). Thus, the contact angle is implicitly imposed by the gradient of phase field at the fluid nodes adjacent to the wall. 


\section{Contact angle hysteresis model}

The wetting boundary condition given in Subsect. II B is suited only for ideal (i.e., rigid, smooth, and chemically homogeneous) solid surfaces with a prescribed contact angle. However, most practical surfaces are rough and heterogeneous to some extent, and the contact angle hysteresis can play a crucial role, e.g. the contact angle hysteresis allows a droplet to be held on a tilted plane and resists the gravity. The contact angle hysteresis is known as a phenomenon in which the contact line remains fixed at a given position, provided that the local contact angle $\theta$ lies within the window of $[36,40,62]$

$$
\theta_{R}<\theta<\theta_{A},
$$

where $\theta_{A}$ and $\theta_{R}$ denote the advancing and receding angles, respectively. On the other hand, if $\theta$ is beyond the hysteresis window, the contact line will move with the moving direction depending on the relative magnitude of $\theta$, $\theta_{A}$ and $\theta_{R}$. Specifically, if $\theta \geq \theta_{A}$, the contact line moves forward; if $\theta \leq \theta_{R}$, the contact line moves backward. To realize the effect of hysteresis, serval numerical strategies have been developed based on the macroscopic multiphase approaches and LBMs [34, 36, 40, 59, 63, 64]. In the present color-fluid LBM, we follow the strategy originally proposed by Ding and Spelt [40] due to its ease in implementation, which can be described as follows. At each time step of simulation, we first keep the values of $\rho_{x, 0}^{N}$ unchanged for all solid nodes (taken from the last time step), and obtain an initial estimate of the local contact angle, $\theta_{0}$, by the use of Eqs. (22) and (23). Then, $\rho_{x, 0}^{N}$ is determined by comparing $\theta_{0}$ with $\theta_{A}$ and $\theta_{R}$ : if $\theta_{0} \geq \theta_{A}$, then set $\theta=\theta_{A}$ and update $\rho_{x, 0}^{N}$ through Eqs. (24) and (25); if $\theta_{0} \leq \theta_{R}$, then set $\theta=\theta_{R}$ and update $\rho_{x, 0}^{N}$ through Eqs. (24) and (25); otherwise, we let $\rho_{x, 0}^{N}$ unchanged.

Note that we here implement the contact angle hysteresis with a given hysteresis window. Due to the microscopic origin of hysteresis, actually, the hysteresis window is determined by the properties of the solid surface in contact with the droplet such as surface roughness and nonuniformity [2,65]. Microscopically, there have been a number of recent works revealing the importance of the contact angle hysteresis and dynamic contact angle [66-68], but the study of the contact angle hysteresis from a viewpoint of microscale is beyond the scope of this work.

\section{RESULTS AND DISCUSSION}

\section{A. Droplet deposited on an ideal substrate with prescribed contact angles}

First, we investigate the equilibrium shapes of a droplet on an ideal substrate to examine the model's capability in simulating prescribed contact angles. Simulations are performed in a $240 \times 160$ lattice domain, and a semi-circular droplet (red fluid) with the radius $R=32$ is initially deposited on the bottom wall. The periodic boundary condition is used in the $x$-direction while the wetting boundary condition, which is given in Subsection II B, is imposed at the top and bottom walls in the $y$-direction. It is known that most existing multiphase/multicomponent LBMs would suffer from numerical instability when the binary fluids have large kinematic viscosity ratio [69]. For instance, the kinematic viscosity ratio is restricted to typically less than 6 in the most-used interparticle-potential LBM [70]. To check whether this also happens to the present model, two different kinematic viscosity ratios $\left(M=\nu_{R} / \nu_{B}\right)$ are investigated: (a) $M=1$ with $\nu_{R}=\nu_{B}=0.35$, and (b) $M=100$ with $\nu_{R}=0.35$ and $\nu_{B}=0.0035$. The other parameters are fixed as $\tilde{\rho}_{R}=\tilde{\rho}_{B}=1$ and $\sigma=0.02$. The velocity and density fields are initialized as $\vec{u}=0$ and

$$
\begin{array}{ll}
\rho_{R}=\tilde{\rho}_{R}, \rho_{B}=0 & \text { if }(x-120)^{2}+(y-0.5)^{2} \leq R^{2} \\
\rho_{R}=0, \rho_{B}=\tilde{\rho}_{B} & \text { otherwise. }
\end{array}
$$

Based on these velocity and density fields, one can determine the initial distribution functions by their equilibrium values, i.e. $f_{i}=f_{i}^{e q}$ and $f_{i}^{k}=\frac{\rho_{k}}{\rho_{R}+\rho_{B}} f_{i}^{e q}$ for $k=R$ or $B$. Each simulation is run until the shape of droplet does not change, i.e., reaching an equilibrium state. Different contact angles can be achieved through adjusting the dimensionless parameter $\Theta_{w}$ according to Eq. (22). Fig. 1 shows equilibrium shapes of the droplet with $\Theta_{w}=\sqrt{3}$, 0 and $-\sqrt{3}$ for the viscosity ratio $M=100$. Their corresponding equilibrium contact angles, calculated from the measured droplet height and base diameter, are $29.68^{\circ}, 89.96^{\circ}$ and $152.68^{\circ}$, respectively. The simulated equilibrium contact angle $\left(\theta^{e q}\right)$ as a function of $\Theta_{w}$ for $M=1$ and $M=100$ is presented in Fig. 2. As expected, the simulations are stable in the entire range of contact angles from $30^{\circ}$ to $150^{\circ}$ for both $M=1$ and $M=100$. Also, it is clearly observed that the simulation results are independent of the viscosity ratio, in good agreement with the theoretical solution, i.e., Eq. (23). A numerical artifact observed in many numerical methods is the presence of spurious velocities at the phase interface. This is also true in our case. Table I shows the maximum spurious velocities $\left(|\vec{u}|_{\text {max }}\right)$ at various $\theta^{e q}$ for $M=1$ and 100, where the values of $|\vec{u}|_{\max }$ are magnified by $10^{4}$ times. For all of the considered contact angles 


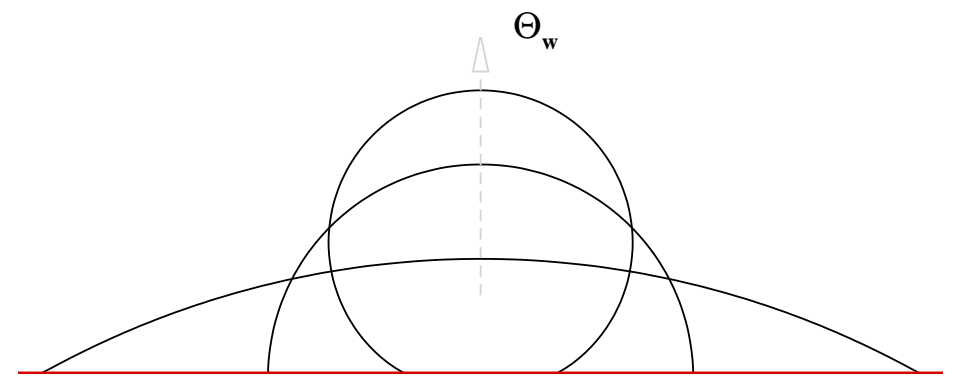

FIG. 1: (Color online) Equilibrium droplet shapes obtained through adjusting the dimensionless parameter $\Theta_{w}$ for $M=100$. The values of $\Theta_{w}$ are taken as $\sqrt{3}, 0$, and $-\sqrt{3}$ along the direction of arrow.

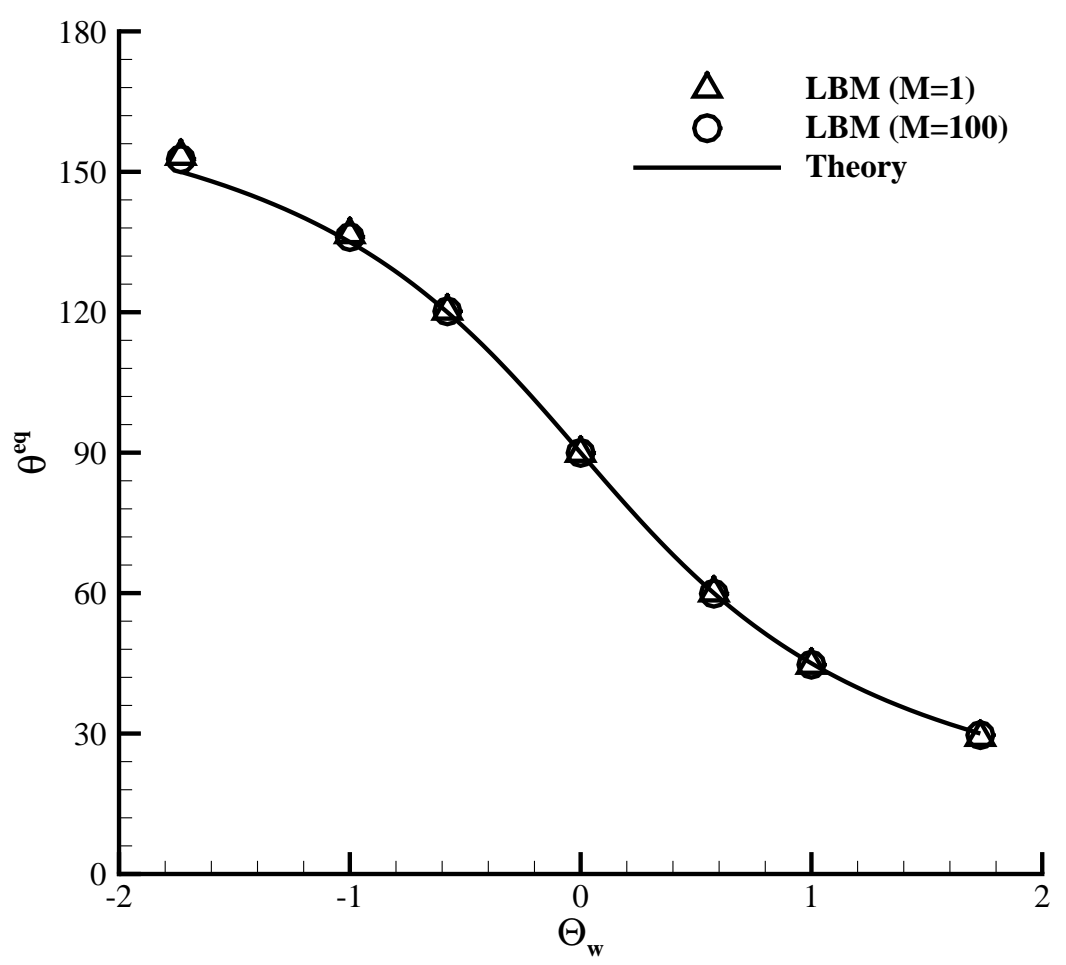

FIG. 2: Contact angle as a function of $\Theta_{w}$ for the viscosity ratios $M=1$ (represented by triangles) and 100 (represented by circles). The solid line represents the theoretical predictions by Eq. (23).

at $M=1$, the maximum spurious velocities are on the order of $10^{-4}$ or even smaller, comparable to those produced by the color-fluid model that used the wetting boundary condition of Ba et al. [61]. It is also seen in Table I that the maximum spurious velocities increase with the viscosity ratio, but most of them are still on the order of $10^{-4}$ for the viscosity ratio as high as 100 .

\section{B. Capillary intrusion}

Capillary intrusion, which originated from the pioneering work of Washburn [71], offers a good benchmarking example for assessing whether a multiphase model is able to simulate moving contact line problems. The velocity of 
TABIF I: The maximum snurious velocities $\left(|\vec{u}|_{\text {man }}\right)$ at various $\theta^{e q}$ for $M=1$ and 100 . All ancles are shown in degrees.

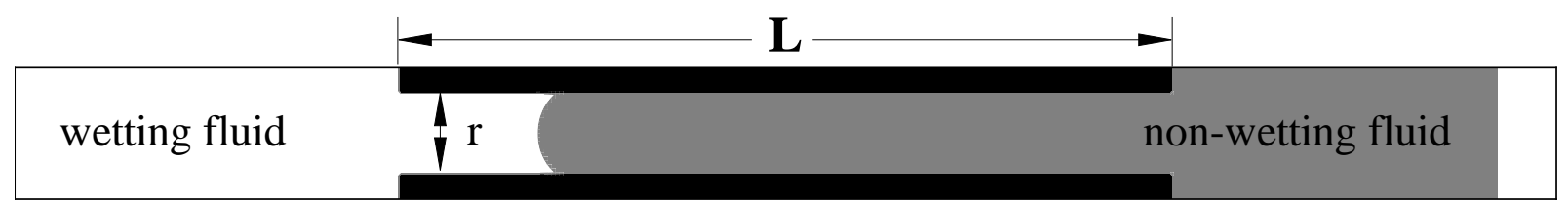

FIG. 3: Simulation setup for capillary intrusion. The portion in the center of the domain is a capillary tube of length $L$ and width $r$. The intruding fluid is wetting with respect to the tube while the defending fluid is non-wetting.

a wetting fluid column intruding a horizontal capillary tube with ideal surfaces, as shown in Fig. 3, is determined by the balance between the pressure difference over the interface, the Laplace pressure, and the viscous drag of the intruding fluid. If the gravity and inertial effects are neglected, this balance can be expressed as [72, 73]

$$
\sigma \cos (\theta)=\frac{6}{r}\left[\mu_{R} \xi+\mu_{B}(L-\xi)\right] \frac{d \xi}{d t}
$$

where $\theta$ is the contact angle, $r$ is the width of capillary tube, $\xi$ is the position of the moving interface with $\xi=0$ at the inlet of capillary tube, and $\mu_{R}$ and $\mu_{B}$ are the dynamic viscosities of the red (wetting) and blue (non-wetting) fluids, respectively. In the analytical derivation, $\theta$ is specified as a wall boundary condition. However, for comparison with simulations or experiments, $\theta$ is better taken as the dynamic contact angle [72, 73]. The system consists of a $400 \times 35$ lattice domain with periodic boundary conditions used in the $x$-direction. In the middle portion, i.e. $100 \leq x \leq 300$, the boundaries of the capillary tube are no-slip and wetting with Eq. (22) imposed on them. The length of the capillary tube is taken as $L=200$ lattices. Outside of the middle portion, the boundary conditions are periodic in the $y$-direction, mimicking an "infinite reservoir". The simulations are run with the parameters $\tilde{\rho}_{R}=\tilde{\rho}_{B}=1$, $\sigma=5 \times 10^{-3}$ and $r=21$ for two different viscosity ratios: (a) $M=1$ and (b) $M=100$. The dimensionless parameter $\Theta_{w}$ is chosen as 1.0 to represent hydrophilic capillary tube for the intruding red fluid. In the fluid domain, the blue fluid is initialized at the position $120 \leq x \leq 375$, and the remaining lattice sites are filled with red fluid. $f_{i}, f_{i}^{R}$ and $f_{i}^{B}$ are then initialized using the zero velocity equilibrium distribution functions. Fig. 4 shows the comparison between our simulation results and the analytical predictions from Eq. (28) for $M=1$ and 100. Note that Eq. (28) is plotted using the dynamic contact angle, measured from our simulations. For $M=1$ and $M=100$, the measured dynamic contact angles are respectively $\theta=46.84^{\circ}$ and $47.11^{\circ}$, which are very close but slightly deviate from their prescribed value $45^{\circ}$. It can be seen that the present color-fluid LBM can predict Eq. (28) pretty well for a broad range of viscosity ratios.

\section{Droplet on a non-ideal substrate subject to a shear flow}

A droplet attached on a nonideal substrate subject to a shear flow is investigated to test the hysteresis behavior of contact angle. We first simulate a droplet pinned on the solid surface due to a large hysteresis window, and the obtained results are compared with the previous numerical results. Then we investigate the influence of hysteresis window and viscosity ratio on droplet behavior at a fixed capillary number.

First, we follow the previous studies $[36,59,64]$ and consider the deformation of a droplet on a non-ideal wall with contact angle hysteresis in a shear flow. This test aims to verify if the contact angle hysteresis is correctly incorporated in our color-fluid LBM. As shown in Fig. 5, the two walls are separated by a distance $H$. The bottom wall is kept stationary, and the top wall moves towards the right with a constant velocity $u_{w}$. Periodic boundary conditions are applied in the horizontal direction. Both fluids have the same density and viscosity, which are given by $\tilde{\rho}_{R}=\tilde{\rho}_{B}=$ 1 , and $\nu_{R}=\nu_{B}=0.2$, respectively. The interfacial tension is set to be $\sigma=5 \times 10^{-3}$. Initially, a droplet of circular segment (red fluid) with the radius $R_{0}$ and contact angle $\theta=60^{\circ}$ is deposited on the bottom wall. Note that one needs to initially specify the phase field of the ghost nodes, i.e. $\rho_{x, 0}^{N}$, when the contact angle hysteresis is considered. $\rho_{x, 0}^{N}$ may keep unchanged and can affect the accuracy of numerical simulations, for example, when the contact lines (or points in 2D) remain pinned. Therefore, the initial values of the phase field must be correctly assigned. In order to minimize spurious velocities caused by the non-smoothness/discontinuity in the phase field, we run the simulation 

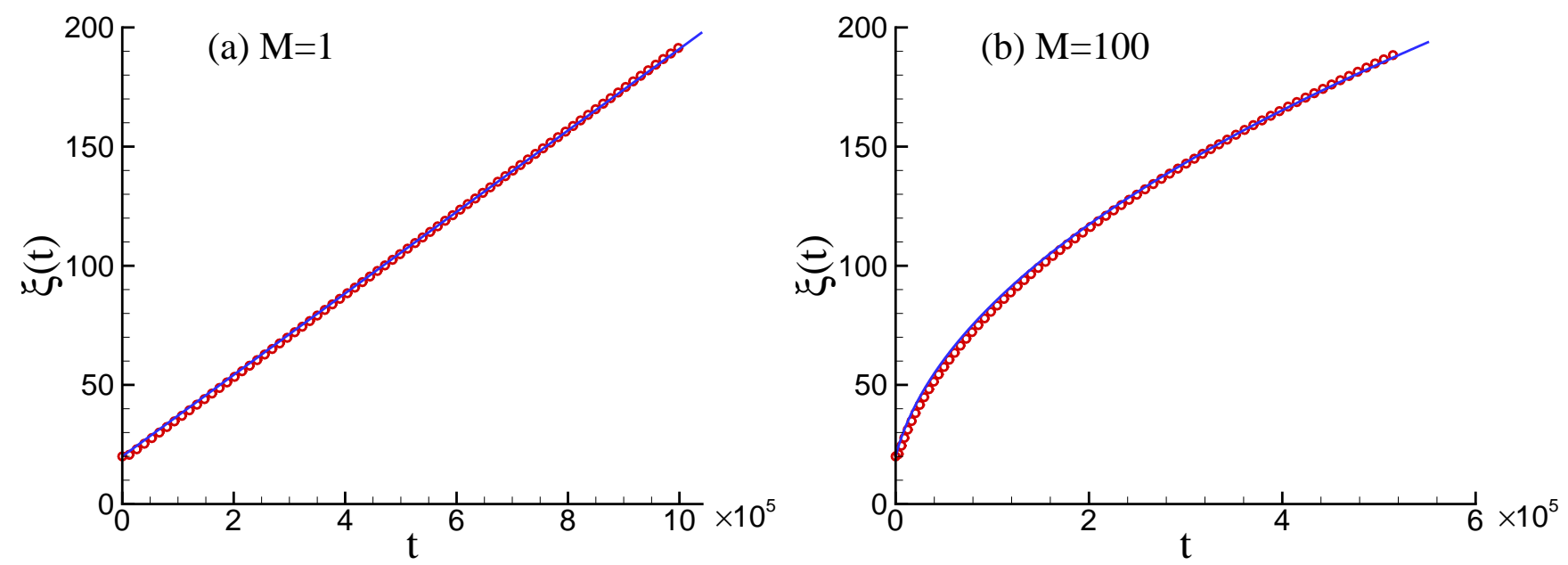

FIG. 4: (Color online) The length of the column of the intruding fluid, $\xi(t)$, versus time for the viscosity ratios of (a) $M=1$ and (b) $M=100$. The (red) open circles represent the simulation results of LBM and the (blue) solid lines are the theoretical predictions from Eq. (28).

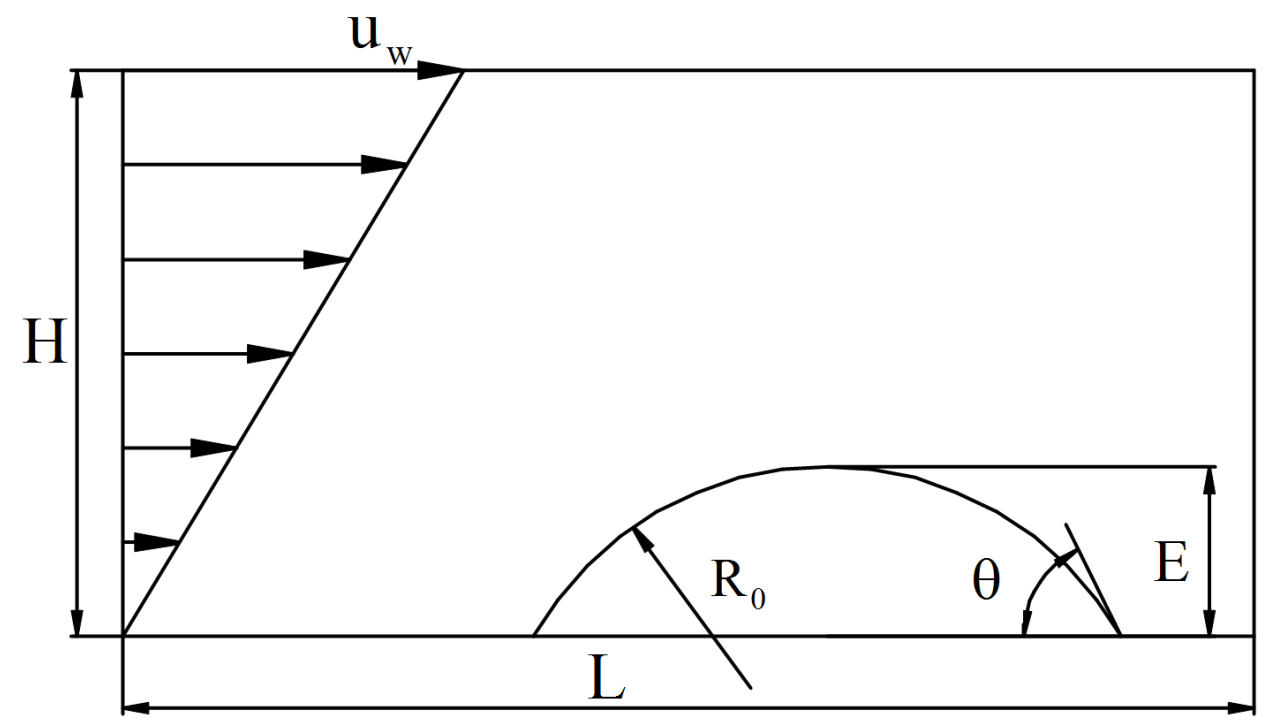

FIG. 5: Schematic diagram of a droplet meniscus on a non-ideal substrate subject to a simple shear flow.

with stationary walls (by setting $u_{w}=0$ as well) and the constant contact angle of $60^{\circ}$ until reaching the steady state, and then assign the steady-state phase field as the initial phase field of the hysteresis simulation. According to the initial phase field $\rho^{N}(\vec{x})$, the initial values of the fluid densities are determined by $\rho_{R}(\vec{x})=\frac{1+\rho^{N}(\vec{x})}{2}$ and $\rho_{B}(\vec{x})=\frac{1-\rho^{N}(\vec{x})}{2}$, and the distribution functions $f_{i}, f_{i}^{R}$ and $f_{i}^{B}$ can be initialized using their corresponding zero velocity equilibrium distributions. The problem is characterized by two important parameters: the scaled droplet area, defined by $A_{d}^{*}=4 A_{d} / H^{2}$ with $A_{d}$ being the droplet area, and the capillary number $C a=\mu_{B} u_{w} E /(\sigma H)$, where $E$ is the initial height of the droplet. We perform the simulations in a $L \times H=500 \times 250$ lattice domain for $C a=0.05$, 0.1 , and 0.15 at a constant $A_{d}^{*}=0.5$. A large hysteresis window $\left(\theta_{R}, \theta_{A}\right)=\left(5^{\circ}, 175^{\circ}\right)[59]$ is imposed through the strategy given in Subsection II C so that the droplet remains pinned on the bottom wall. This allows us to compare our results with those obtained by Schleizer and Bonnecaze [74], where a boundary element method was used.

Fig. 6 compares the simulated equilibrium droplet shapes with the numerical results of Schleizer and Bonnecaze [74] for $C a=0.05,0.1$, and 0.15 at $A_{d}^{*}=0.5$. As $C a$ increases, the droplet deforms more significantly, and the difference between the downstream and upstream contact angles increases. For all of the capillary numbers, the simulated droplet shapes (represented by solid lines) agree well with the results of Schleizer and Bonnecaze (represented by dashed lines), revealing good accuracy of the present model for handling contact angle hysteresis.

Next, we investigate the deformation, breakup and migration of the droplet for different hysteresis windows at a 

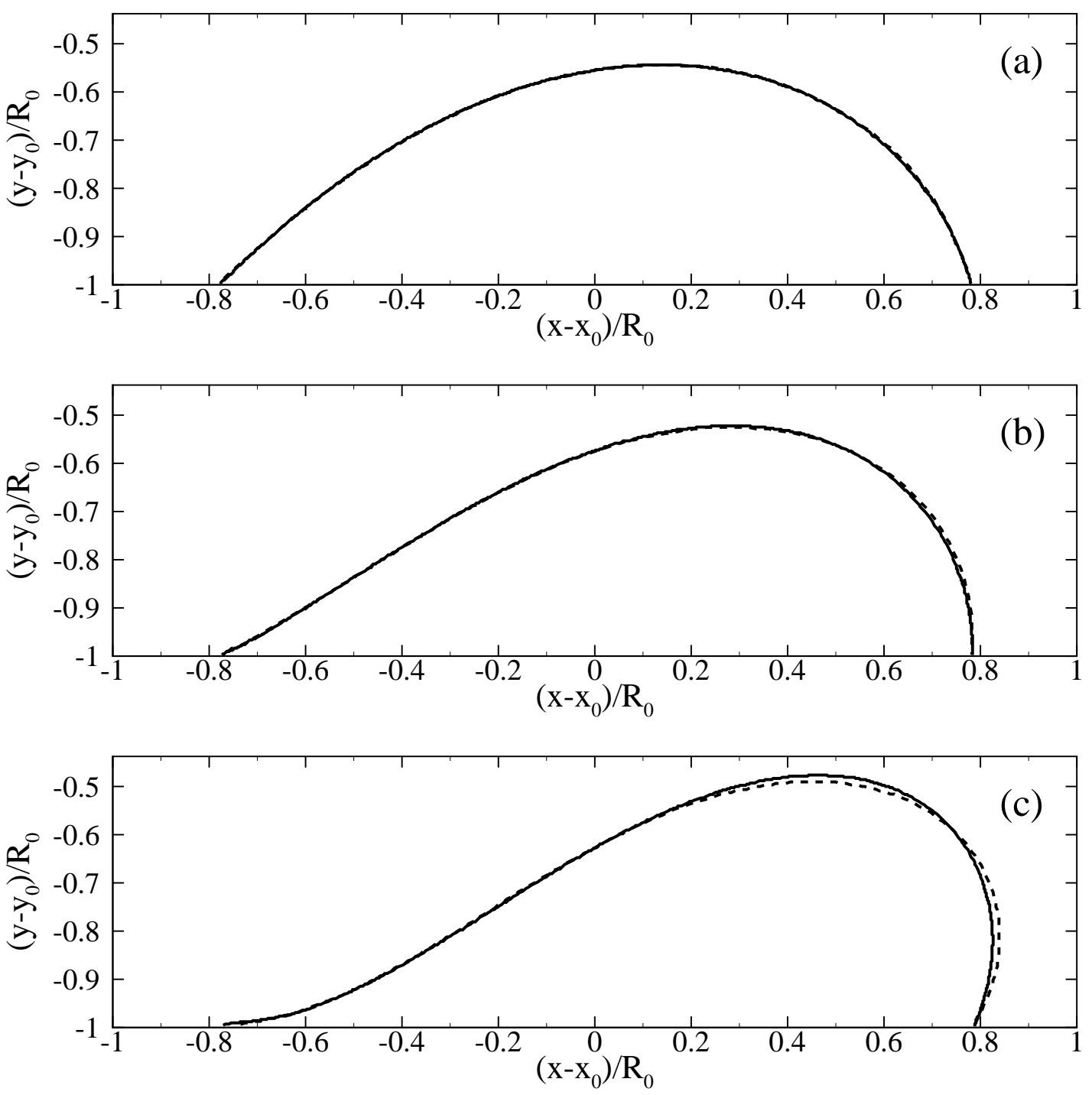

FIG. 6: Comparison of the simulated equilibrium droplet shapes with the numerical results of Schleizer and Bonnecaze [74] for $C a=0.05,0.1$, and 0.15 at $A_{d}^{*}=0.5$. The solid lines are the present LBM simulation results, while the dashed lines are the results of Schleizer and Bonnecaze. The $x$ - and $y$-coordinates relative to the center of computational domain $\left(x_{0}, y_{0}\right)$ are normalized by $R_{0}$.

fixed capillary number and viscosity ratio. A semicircular droplet of radius $R_{0}=64$ is initially placed on the bottom wall in a $1024 \times 128$ lattice domain. Both fluids are assumed to have equal density and kinematic viscosity, i.e., $\tilde{\rho}_{R}=\tilde{\rho}_{B}=1$ and $\nu_{R}=\nu_{B}=0.25$, and the interfacial tension is set as $\sigma=10^{-3}$. We use the same initialization strategy and boundary conditions described above (except that the initial contact angle $\theta=90^{\circ}$ ), and choose the moving velocity of the top wall $u_{w}=2.56 \times 10^{-3}$ such that $C a=0.32$ and $R e=u_{w} R_{0}^{2} /\left(H \nu_{B}\right)=0.328$. Besides, the hysteresis windows are chosen as $\left(0^{\circ}, 180^{\circ}\right),\left(0^{\circ}, 110^{\circ}\right),\left(70^{\circ}, 180^{\circ}\right)$, and $\left(70^{\circ}, 110^{\circ}\right)$, which have been used recently by Wang et al. [64] for qualitatively testing their model.

Fig. 7 shows the snapshots of droplet motion for the hysteresis windows of $\left(0^{\circ}, 180^{\circ}\right),\left(0^{\circ}, 110^{\circ}\right),\left(70^{\circ}, 180^{\circ}\right)$, and $\left(70^{\circ}, 110^{\circ}\right)$ at $M=1$ and $C a=0.32$, where the dimensionless time is defined as $t^{*}=\gamma t$ with the shear rate $\gamma=u_{w} / H$. In the early stages, the droplet deforms and/or moves downstream on the bottom wall. As expected, one can observe four typical motion modes of the contact points (note that we use the contact point instead of the contact line in twodimensional problems) due to four hysteresis windows. For $\left(\theta_{R}, \theta_{A}\right)=\left(0^{\circ}, 180^{\circ}\right)$, both the upstream and downstream contact angles cannot go outside of the hysteresis window, so both contact points always remain motionless on the solid surface. For $\left(\theta_{R}, \theta_{A}\right)=\left(0^{\circ}, 110^{\circ}\right)$, due to strong shear stresses from the carrier fluid the upstream contact angle is decreased from the right angle to acute angles, which are obviously in the range $\left(0^{\circ}, 110^{\circ}\right)$, so the upstream contact point remains fixed. For the downstream contact point, it is pinned initially when the instantaneous contact 

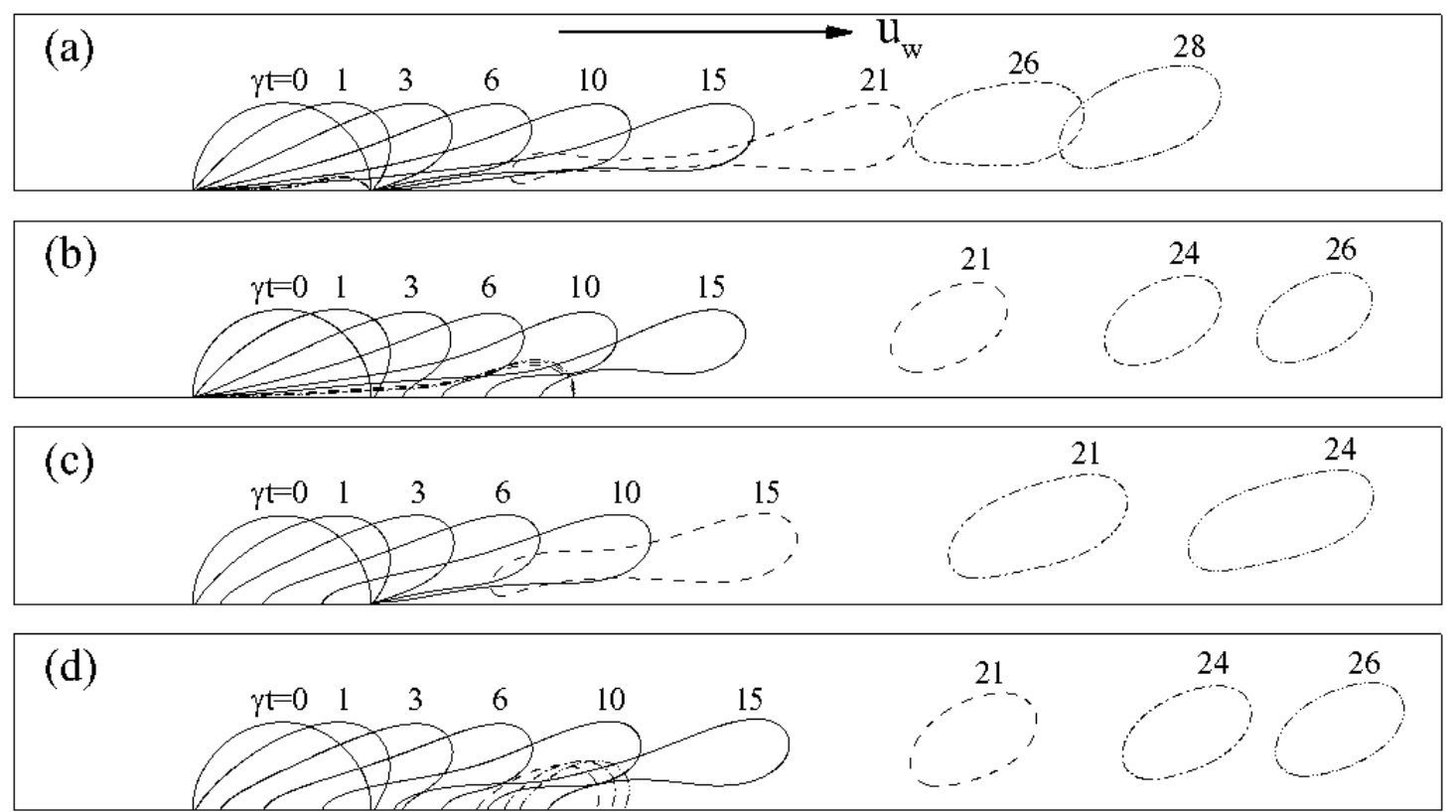

FIG. 7: Snapshots of droplet motion for the hysteresis windows of $\left(0^{\circ}, 180^{\circ}\right),\left(0^{\circ}, 110^{\circ}\right),\left(70^{\circ}, 180^{\circ}\right)$, and $\left(70^{\circ}, 110^{\circ}\right)$ at $M=1$ and $C a=0.32$. Note that the droplet profiles are represented successively by the dashed lines, dash-dot lines and dash-dot-dot lines at the final three instants after the breakup or detachment from the wall.

angle is less than $110^{\circ}$, and later it moves downstream as long as the contact angle becomes larger than $110^{\circ}$. For $\left(\theta_{R}, \theta_{A}\right)=\left(70^{\circ}, 180^{\circ}\right)$, the downstream contact angle keeps increasing continuously, but it stays in the range of hysteresis window at all times, resulting in the downstream contact point immobilized. For the upstream contact point, during the initial short time (typically when $\gamma t \leq 1$ ) it is pinned because the instantaneous contact angle is larger than $70^{\circ}$, and later it progresses downstream along the surface when the contact angle is less than $70^{\circ}$. For $\left(\theta_{R}, \theta_{A}\right)=\left(70^{\circ}, 110^{\circ}\right)$, the droplet keeps pinned and deforms continuously until the upstream and downstream contact angles reach their hysteresis limits, and then starts to slip over the wall. These results are in qualitative agreement with the previous computations of Wang et al. [64], which is built upon the mean-field theory model. As the droplet deforms and/or the contact points (which might be the upstream contact point only, the downstream contact point only, or both) progress downstream further, one can observe two different dynamical behaviors of droplet in the late stages. Specifically, if $\left(\theta_{R}, \theta_{A}\right)=\left(70^{\circ}, 180^{\circ}\right)$, the droplet departs entirely from the solid surface and finally moves at a constant velocity in the carrier fluid free of wetting effects; otherwise, the droplet breaks up into two separate parts: one moves fast as it breaks away from the solid surface, while the other adheres to the solid surface with its shape, size and motion state depending on the hysteresis window. Clearly, the adhering part eventually remains immobilized for $\left(\theta_{R}, \theta_{A}\right)=\left(0^{\circ}, 180^{\circ}\right)$ and $\left(0^{\circ}, 110^{\circ}\right)$, but slides over the surface at a constant, relatively low velocity for $\left(\theta_{R}, \theta_{A}\right)=\left(70^{\circ}, 110^{\circ}\right)$.

In addition, we also simulate the deformation, breakup and migration of the droplet at various hysteresis windows for the viscosity ratio of 0.01 , and the obtained results will be compared with the case of $M=1$ (see Fig. 7 ) to show the influence of viscosity ratio on the hysteresis behavior. All the simulation parameters are kept the same as in the case of $M=1$ except that the viscosity of the dispersed phase (red fluid) is changed to be $\nu_{R}=0.0025$. Fig. 8 illustrates the time evolution of droplet shapes for the hysteresis windows of $\left(0^{\circ}, 180^{\circ}\right),\left(0^{\circ}, 110^{\circ}\right),\left(70^{\circ}, 180^{\circ}\right)$, and $\left(70^{\circ}, 110^{\circ}\right)$ at $M=0.01$ and $C a=0.32$. For $\left(\theta_{R}, \theta_{A}\right)=\left(0^{\circ}, 180^{\circ}\right)$, both the upstream and downstream contact points remains pinned on the bottom wall at all times. From its initial semi-circular shape, the droplet gradually deforms and finally reaches a stationary shape (represented by the red bold lines in Fig. 8(a)). In contrast, for $M=1$ the droplet continuously deforms and eventually breaks up into two parts, one of which remains pinned on the bottom wall forever and the other moves downstream in the carrier fluid (see Fig. $7(\mathrm{a}))$. For $\left(\theta_{R}, \theta_{A}\right)=\left(0^{\circ}, 110^{\circ}\right)$, the upstream contact point cannot move due to the corresponding contact angle always within the hysteresis window. The downstream contact point evolves in a relatively complex manner: first, it is pinned; then, it moves downstream as the instantaneous contact angle is larger than $110^{\circ}$; finally, it is pinned again and the entire droplet reaches a steady state. This behavior is evidently different from the previous observation that the downstream contact point first undergoes a short "pinning" process, and then keeps moving on the wall until the droplet is divided into two 

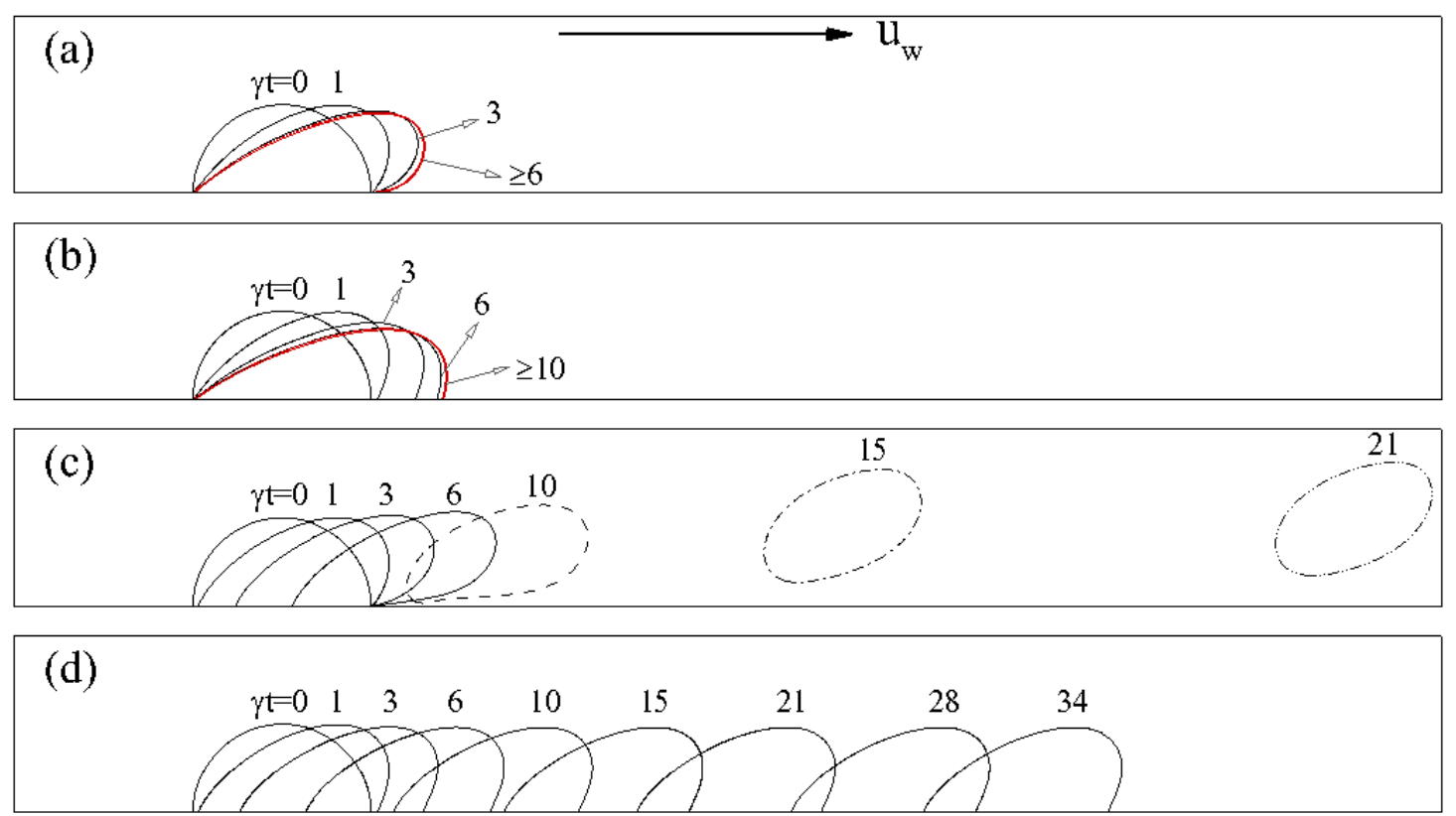

FIG. 8: (Color Online) Snapshots of droplet motion for the hysteresis windows of $\left(0^{\circ}, 180^{\circ}\right),\left(0^{\circ}, 110^{\circ}\right),\left(70^{\circ}, 180^{\circ}\right)$, and $\left(70^{\circ}, 110^{\circ}\right)$ at $M=0.01$ and $C a=0.32$. In (a) and (b), the droplet profiles in the steady state are represented by the (red) bold lines, while in (c) the droplet profiles are represented successively by the dashed lines, dash-dot lines and dash-dot-dot lines at the final three instants after the detachment from the wall.
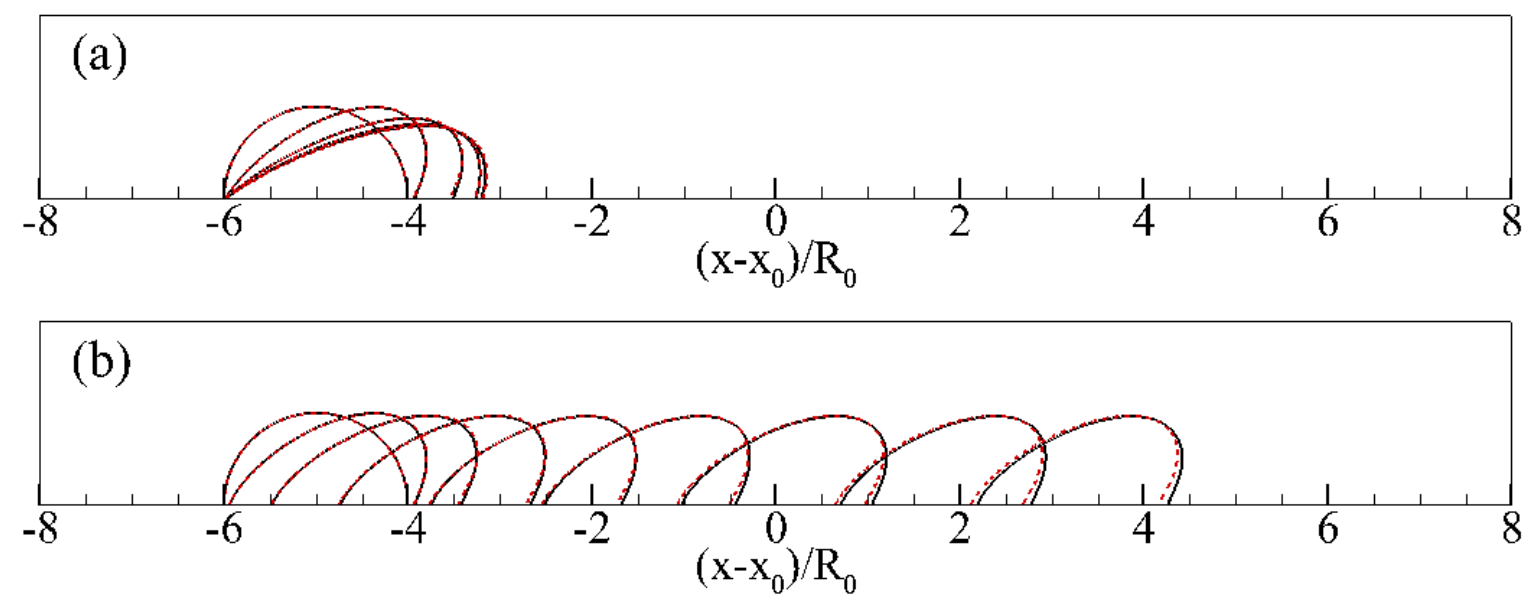

FIG. 9: (Color Online) Snapshots of droplet motion in the coarse and fine grids for the hysteresis windows of $(\mathrm{a})\left(0^{\circ}, 110^{\circ}\right)$ and (b) $\left(70^{\circ}, 110^{\circ}\right)$ at $M=0.01$ and $C a=0.32$. Note that the droplet profiles in the coarse and fine grids are shown by the black solid lines and the red dashed lines, respectively. The $x$ - and $y$-coordinates relative to the center of computational domain $\left(x_{0}, y_{0}\right)$ are normalized by $R_{0}$.

small ones, as illustrated in Fig. $7(\mathrm{~b})$. For $\left(\theta_{R}, \theta_{A}\right)=\left(70^{\circ}, 180^{\circ}\right)$, the droplet motion (including the motion of both contact points) exhibits similar behavior to those observed in the case of $M=1$; nevertheless, there exists quantitative difference in the droplet shape and terminal velocity, as well as the velocity of moving contact point for both $M=0.01$ and $M=1$. For $\left(\theta_{R}, \theta_{A}\right)=\left(70^{\circ}, 110^{\circ}\right)$, in the early stages the motion of contact points is analogous to the previous observation of $M=1$; whereas in the late stages the droplet reaches a steady shape and slides over the bottom wall at a constant velocity, differing significantly from the pinch-off behavior observed in the case of $M=1$. Thus, it can be concluded that the viscosity ratio plays an extremely important role in determining the dynamical behavior of a droplet when the contact angle hysteresis is taken into account.

Grid convergence, also known as grid independence, is investigated by comparing the simulation results in the coarse and fine grids, which have dimensions $1024 \times 128$ and $2048 \times 256$, respectively. Two representative cases are 


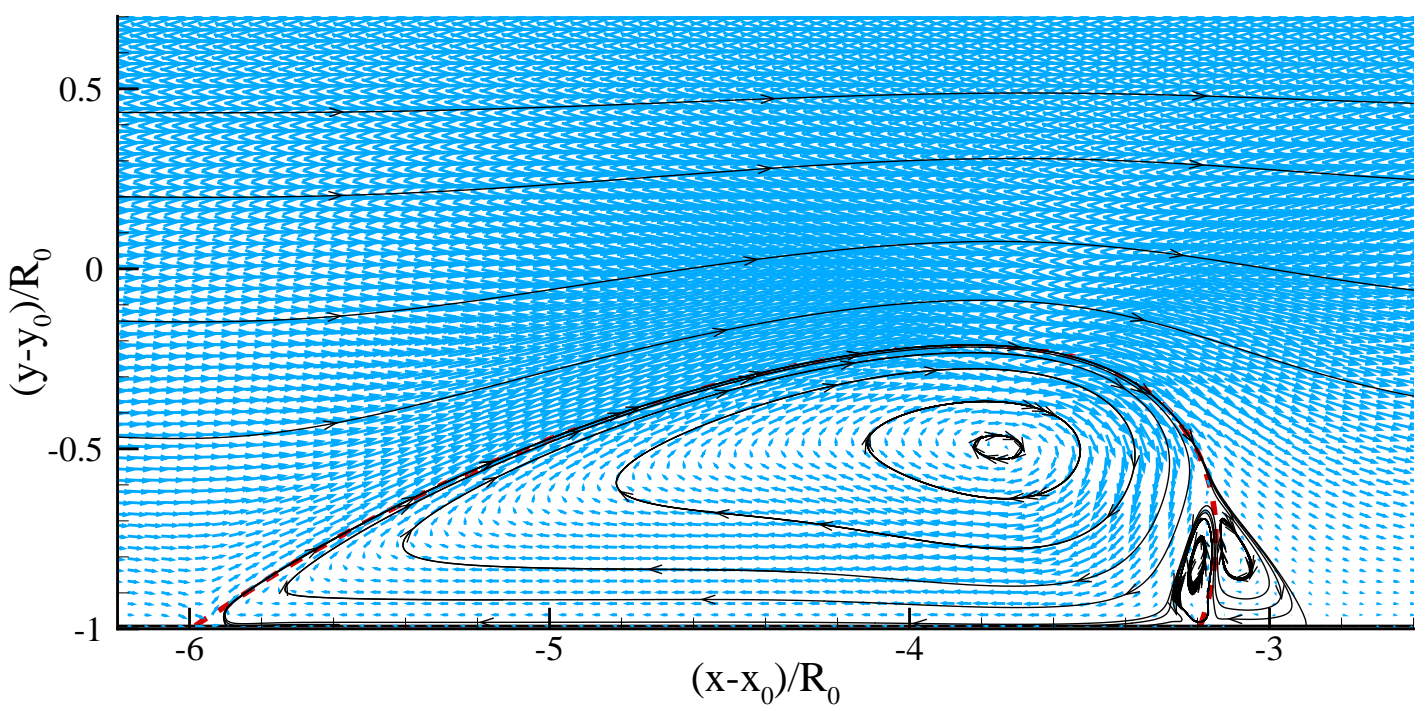

(a)

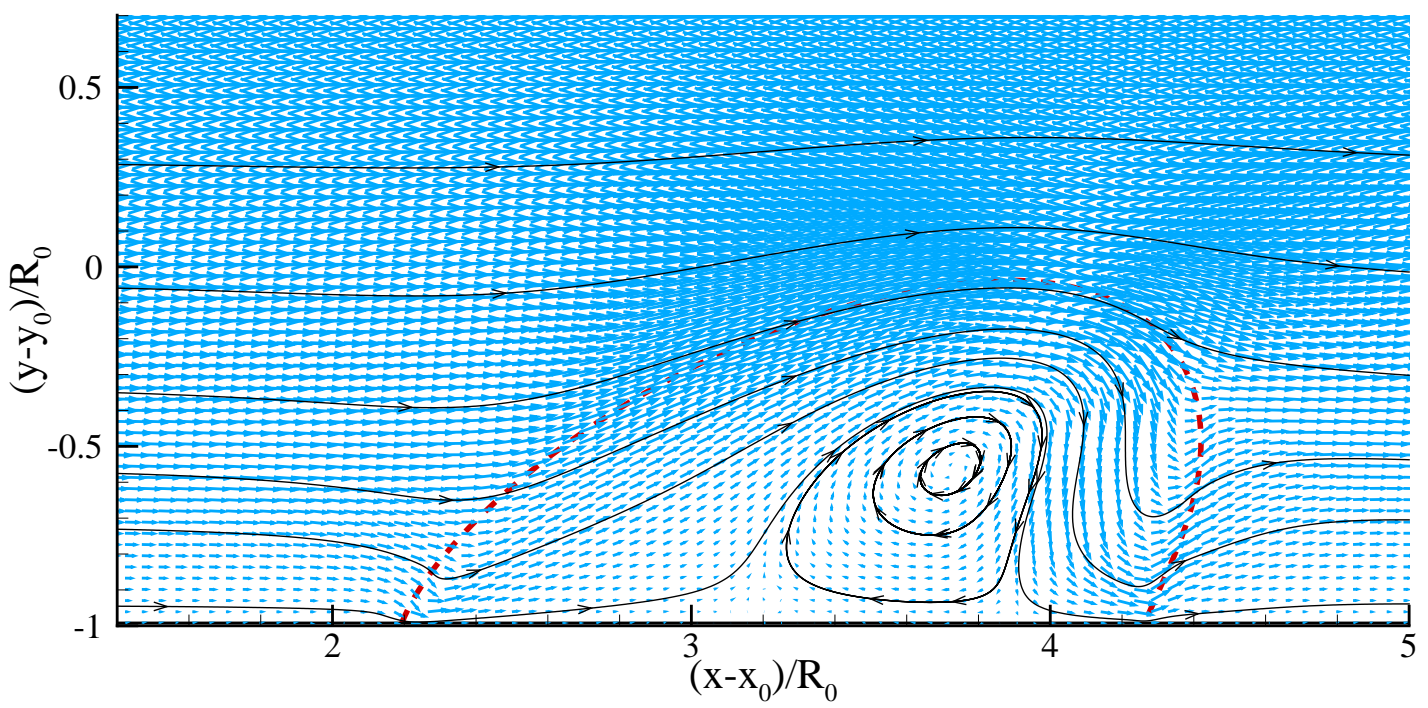

(b)

FIG. 10: (Color online) The velocity vectors and streamlines around the droplet for (a) $\left(\theta_{R}, \theta_{A}\right)=\left(0^{\circ}, 110^{\circ}\right)$ in the steady state and (b) $\left(\theta_{R}, \theta_{A}\right)=\left(70^{\circ}, 110^{\circ}\right)$ at $\gamma t=34$ in the computational domain of $1024 \times 128$ grids. Note that the droplet profiles are represented by the red dashed lines, and the $x$ - and $y$-coordinates relative to the center of computational domain $\left(x_{0}, y_{0}\right)$ are normalized by $R_{0}$.

simulated: one with the hysteresis window of $\left(0^{\circ}, 110^{\circ}\right)$, and the other with $\left(70^{\circ}, 110^{\circ}\right)$. Both cases have the same viscosity ratio, capillary number and Reynolds number, i.e., $M=0.01, C a=0.32$ and $R e=0.328$. Fig. 9 gives the comparison of simulation results between the coarse grid and the fine grid for (a) $\left(\theta_{R}, \theta_{A}\right)=\left(0^{\circ}, 110^{\circ}\right)$ and $(\mathrm{b})$ $\left(\theta_{R}, \theta_{A}\right)=\left(70^{\circ}, 110^{\circ}\right)$. Note in Fig. 9 that the simulation results in the coarse grid have been plotted previously in Fig. 8(b) and (d). It is clearly seen that the grid refinement only has a slight effect on the simulation results, indicating that the coarse grid with the domain size of $1024 \times 128$ grids can provide acceptable numerical accuracy. Since the interface thickness decreases with the grid refinement, the slight effect of the grid refinement on the simulation results also indicates that a further decrease in the interface thickness almost has no effect on the simulation results, i.e. approaching the sharp-interface limit. Fig. 10(a) and (b) depict the velocity vectors and streamlines around the droplet interface in the coarse grid for $\left(\theta_{R}, \theta_{A}\right)=\left(0^{\circ}, 110^{\circ}\right)$ in the steady state and for $\left(\theta_{R}, \theta_{A}\right)=\left(70^{\circ}, 110^{\circ}\right)$ at $\gamma t=34$. For $\left(\theta_{R}, \theta_{A}\right)=\left(0^{\circ}, 110^{\circ}\right)$, we can observe that two unequal-sized vortices are fully filled with the droplet inside, implying that there is not momentum exchange between the droplet and the carrier fluid; whereas for $\left(\theta_{R}, \theta_{A}\right)=\left(70^{\circ}, 110^{\circ}\right)$, some streamlines can pass through the droplet interface, suggesting that there exists momentum exchange between 


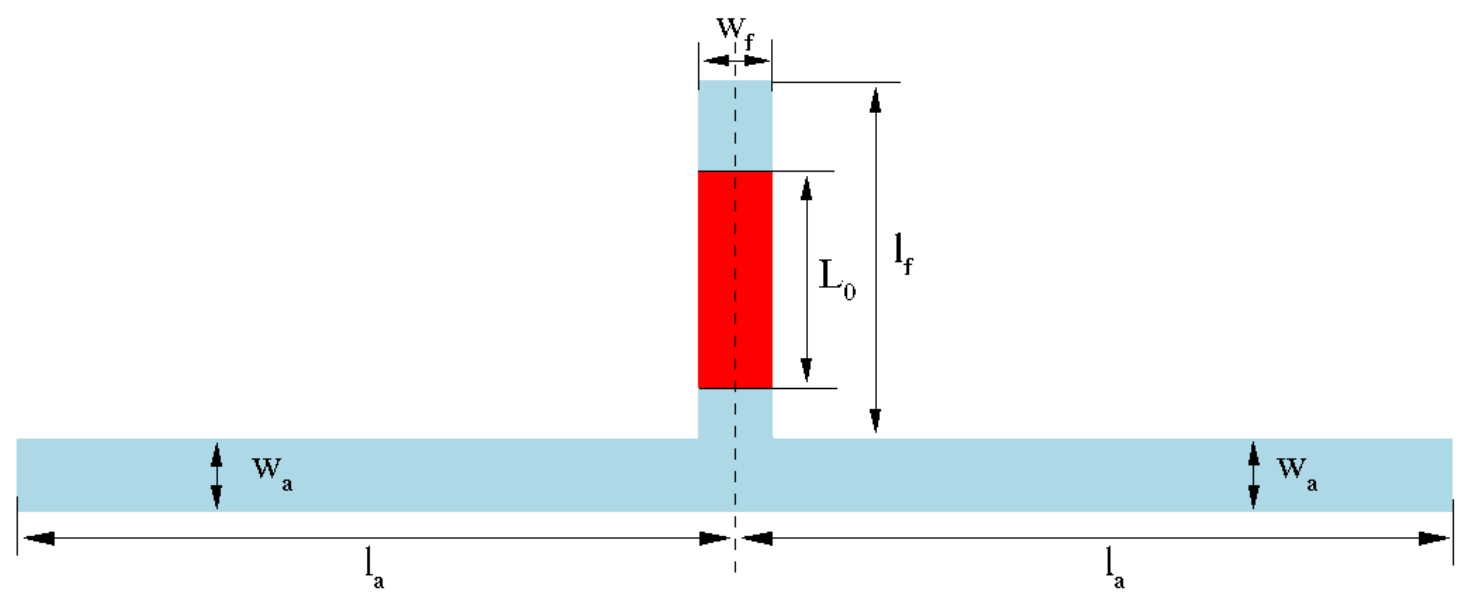

FIG. 11: (Color Online) Schematic of T-junction geometry.

the droplet and the carrier fluid. These behaviors of streamlines are consistent with the motion of droplet over the solid surface. Also, we do not see unexpected vortices or weird variations of velocity in the vicinity of interface, in particular around the contact points, indicating that unphysical spurious velocities are effectively suppressed in the present LBM even when the contact angle hysteresis is considered.

\section{Droplet breakup process at a microfluidic T-junction}

As stated above, the color-fluid model proposed by Ba et al. [34] suffers from the difficulty of handling contact line dynamics with complex solid boundaries. In order to demonstrate that such a difficulty can be greatly overcome in the present model, we use it to simulate the droplet breakup process at a microfluidic T-junction, where the convex corner is also encountered in addition to the straight walls. We consider a red droplet of initial length $L_{0}=120$ that flows from a channel of width $w_{f}=40$ and length $l_{f}=200$ into a T-junction with two equal arms of the same width $w_{a}=40$ and length $l_{a}=400$ (see Fig. 11). The droplet is carried downstream by the blue fluid, which flows through the feed branch of the T-junction with the mean velocity $U$. The channel walls in the right half of the geometry are ideal with constant contact angle of $135^{\circ}$, while the rest of channel walls are non-ideal with the hysteresis window of $\left(120^{\circ}, 150^{\circ}\right)$. For the sake of simplicity, both fluids are assumed to have equal densities, i.e. $\tilde{\rho}_{R}=\tilde{\rho}_{B}=1$, and the interfacial tension is fixed at 0.02. Considering that the flow in the microchannel is typically laminar, the Reynolds number $\left(R e=\tilde{\rho}_{B} U w_{f} / \mu_{B}\right)$ is selected as 0.4 without loss of generality. The capillary number, which describes the relative importance of viscosity to the interfacial tension and is defined by $C a=U \mu_{B} / \sigma$, is commonly used to characterize the droplet breakup process, and its value is $5 \times 10^{-3}$. In this subsection, we will examine whether and how the contact angle hysteresis and the viscosity ratio influence the dynamics of droplet breakup. A parabolic velocity profile and a constant pressure are specified respectively, at the inlet and outlets using the non-equilibrium bounce-back scheme [75]. The no-slip boundary condition is applied at the wall surfaces. For the straight walls, the surface wettability is introduced directly following the method described in Sects. II B and II C; whereas for the convex corners, the following method is adopted to determine $\rho^{N}(x, y)$, where $(x, y)$ represents the solid node most adjacent to the convex corner:

1. Suppose that $(x, y)$ is the ghost node of the horizontal wall, and use the method described in Sects. IIB and II C to determine the phase field at this node, denoted by $\rho_{h}^{N}$;

2. Suppose that $(x, y)$ is the ghost node of the vertical wall, and use the method described in Sects. II B and II C to determine the phase field at this node, denoted by $\rho_{v}^{N}$;

3. Update $\rho^{N}(x, y)$ using the average of $\rho_{h}^{N}$ and $\rho_{v}^{N}$, i.e. $\rho^{N}(x, y)=\left(\rho_{h}^{N}+\rho_{v}^{N}\right) / 2$.

We run the simulations at three different viscosity ratios, i.e. $M=3,1$ and 0.1 , which are achieved by varying solely the viscosity of the red fluid. Each simulation is initialized with the density field

$$
\begin{array}{ll}
\rho_{R}=\tilde{\rho}_{R}, \rho_{B}=0 & \text { if } 70 \leq y \leq 190 \\
\rho_{R}=0, \rho_{B}=\tilde{\rho}_{B} & \text { otherwise, }
\end{array}
$$




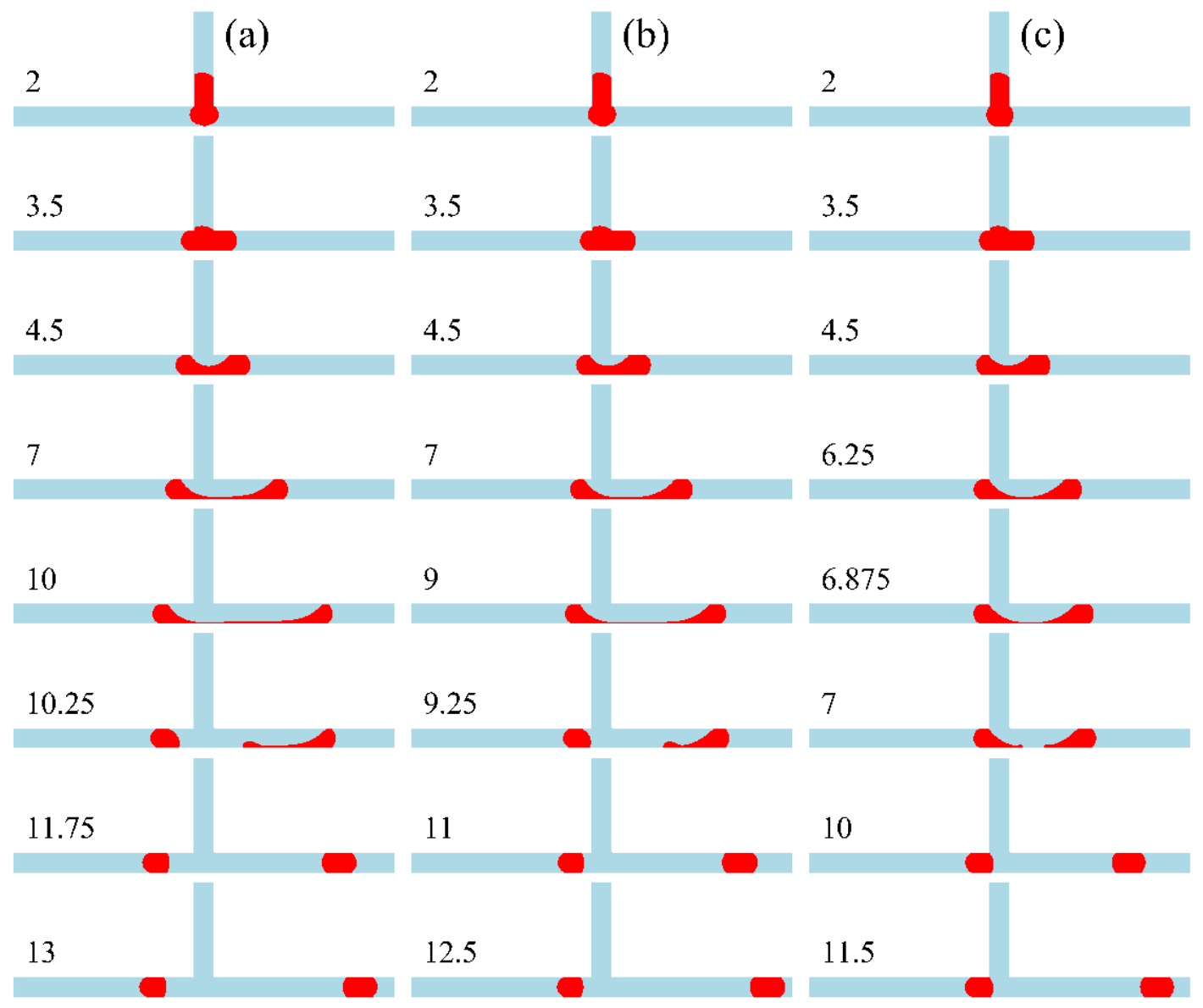

FIG. 12: (Color Online) Snapshots of droplet breakup in the 'non-ideal' T-junction for $C a=5 \times 10^{-3}$ and $R e=0.4$ at different viscosity ratios: (a) $M=3$, (b) $M=1$ and (c) $M=0.1$. For the T-junction, the right half walls are assumed to be ideal with the constant contact angle of $135^{\circ}$, while the remaining walls are non-ideal with the hysteresis window of $\left(120^{\circ}, 150^{\circ}\right)$. The dimensionless time is defined by $t^{*}=U t / w_{f}$. Note that the droplet and carrier fluid are shown in red and blue, respectively.

and zero velocity field everywhere except at the inlet, where the parabolic velocity profile is prescribed. By means of the density and velocity fields, the initial distribution functions are calculated as $f_{i}=f_{i}^{e q}$ and $f_{i}^{k}=\frac{\rho_{k}}{\rho_{R}+\rho_{B}} f_{i}^{e q}(k=R$ or $B)$.

Fig. 12 shows the snapshots of droplet breakup in the T-junction at three different viscosity ratios: (a) $M=3$, (b) $M=1$ and (c) $M=0.1$. Note in Fig. 12 that the dimensionless time is defined by $t^{*}=U t / w_{f}$. The droplet first moves downward and its head touches the bottom wall of the horizontal channel at around $t^{*}=2$. Later, the droplet continues to fill the junction until completely entering the junction at $t^{*}=3.5$. During this time, the droplet invades the left and right branches and advances inside, but the droplet advances more slowly in the left branch due to the contact angle hysteresis. Soon after $t^{*}=3.5$, a bent neck is formed under the squeezing of the carrier fluid flowing into the T-junction. The span of the bent neck is gradually expanded, and the expansion mainly occurs in the right branch of the horizontal channel. This suggests that the carrier fluid mostly flows into the right branch as a result of the interface retardation in the left branch. The droplet eventually pinches off at its minimum width and is asymmetrically split into two daughter droplets. It is clearly seen that the daughter droplet in the branch with non-ideal surfaces is smaller than the other one. We interestingly note that, during the entire breakup process the droplet tips always keep touching with the channel walls, evincing that the horizontal channels are continuously obstructed by droplets. This is known as breakup with permanent obstruction (POB), which has been observed both experimentally and numerically in microfluidic T-junctions [76-78].

Even though the obtained results are qualitatively similar for different viscosity ratios, as shown above, there do exist some quantitative differences in the droplet behavior. For instance, decreasing the viscosity ratio can cause the droplet to break up earlier and decreases the maximum length that the droplet could reach before the breakup. In late stages, albeit the daughter droplet in the right branch of the horizontal channel keeps moving towards the right, the daughter droplet in the left branch exhibits different states of motion. Specifically, at high viscosity ratio, i.e. 

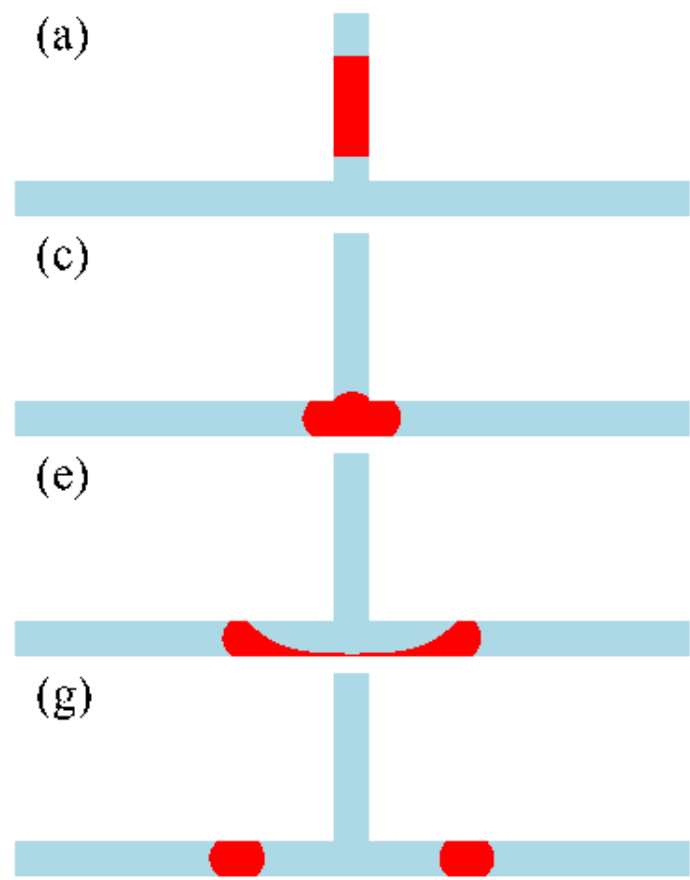
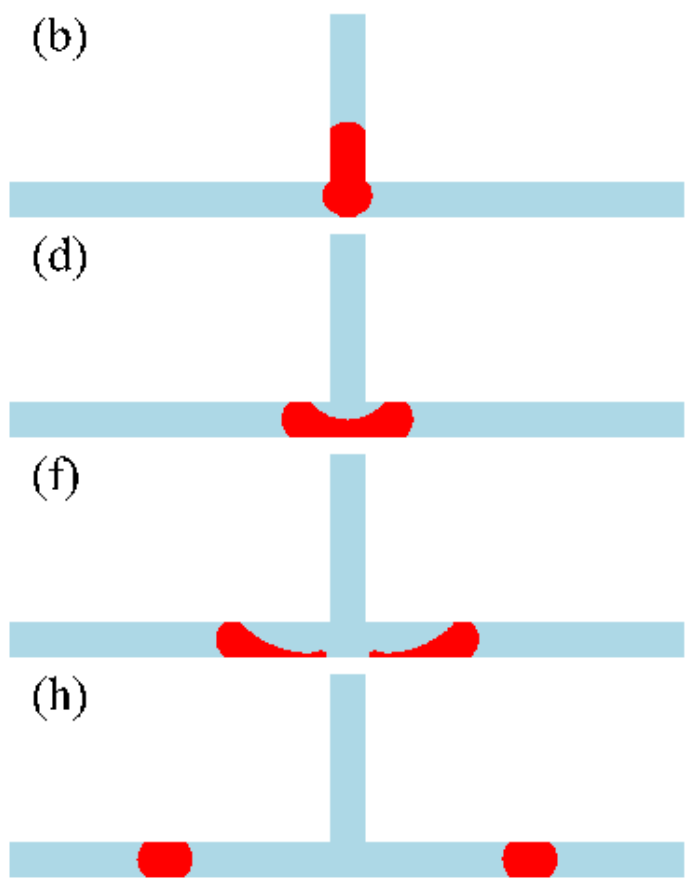

FIG. 13: (Color Online) Snapshots of droplet breakup in the 'ideal' T-junction with the contact angle of $135^{\circ}$ for $M=1$, $C a=5 \times 10^{-3}$ and $R e=0.4$. The snapshots are taken at the times: (a) $t^{*}=0$, (b) $t^{*}=2$, (c) $t^{*}=3.5,(\mathrm{~d}) t^{*}=4.5,(\mathrm{e})$ $t^{*}=8.25,(\mathrm{f}) t^{*}=8.375,(\mathrm{~g}) t^{*}=9,(\mathrm{~h}) t^{*}=13$. Note that the droplet and carrier fluid are shown in red and blue, respectively.

$M=3$, the daughter droplet keeps migrating through the left branch channel, but at a velocity much lower than the right one (see $t^{*}=10.25,11.75$ and 13 in Fig. $12(\mathrm{a})$; at medium and low viscosity ratios, i.e. $M=1$ and 0.1 , the daughter droplet gets pinned in the left branch, but its rest position depends on $M$ : the droplet remains ultimately pinned at a certain distance from the T-junction and at the junction corner for $M=1$ and $M=0.1$, respectively. These quantitative differences in the droplet behavior indicate that the contact angle hysteresis can be strengthened with the decrease of $M$, consistent with the finding in Subsect. III C. In addition, we also quantify the length of the daughter droplet $\left(L_{r}\right)$ in the right branch channel and find that the values of $L_{r}$ are $73.2,74.6$ and 70.7 for $M=3,1$ and 0.1 , respectively. Obviously, $L_{r}$ varies non-monotonously with the decrease of viscosity ratio, but the variations are not so noticeable in the cases under consideration.

Finally, we simulate the droplet breakup process at the T-junction with all the parameters unchanged except for the hysteresis window which is now $\left(135^{\circ}, 135^{\circ}\right)$. Such a hysteresis window implies that the entire channel walls are ideal with a constant contact angle of $135^{\circ}$. Fig. 13 depicts the snapshots of droplet breakup in the T-junction for $\left(\theta_{R}, \theta_{A}\right)=\left(135^{\circ}, 135^{\circ}\right)$ at $M=1$. Compared to the case of $\left(\theta_{R}, \theta_{A}\right)=\left(120^{\circ}, 150^{\circ}\right)$ (see Fig. $\left.12(\mathrm{~b})\right)$, the droplet pinches off at an earlier time, i.e. $t^{*}=8.375$, and the maximum length that the droplet can reach before the pinch-off is smaller: specifically, the values of the maximum length are 306 and 337 for $\left(\theta_{R}, \theta_{A}\right)=\left(135^{\circ}, 135^{\circ}\right)$ and $\left(120^{\circ}, 150^{\circ}\right)$, respectively. Due to the symmetry of the channel geometry and the imposed surface wettability, the droplet symmetrically moves and deforms, eventually breaking up into two equal-sized daughter droplets. The two daughter droplets then move at the same speed towards the outlets. These droplet behaviors are evidently different from the previous observations in the case of $\left(\theta_{R}, \theta_{A}\right)=\left(120^{\circ}, 150^{\circ}\right)$, suggesting that the hysteresis window is one of important factors characterizing the droplet breakup process.

\section{CONCLUSIONS}

In this paper, a multiphase LBM is developed to simulate contact-line dynamics, particularly with contact angle hysteresis, for a broad range of viscosity ratios. In this method, the immiscible two-phase flows are described by a color-fluid LBM, originally proposed by Halliday and his coworkers [20, 42, 43]. Instead of using the original BGK approximation, the color-fluid LBM is implemented in the framework of MRT, which can enhance the numerical stability for solving binary fluids with larger viscosity difference and suppress spurious currents arising at the interface. The specified hysteresis of contact angle is realized by utilizing the strategy of Ding and Spelt [40], and the resulting 
contact angle is enforced through the geometrical formulation proposed in Ref. [41], with implementation following Huang et al. [59]. The color-fluid LBM is first validated by simulations of static contact angle and dynamic capillary intrusion on the ideal surfaces for the viscosity ratios of 1 and 100. It is then used to simulate the dynamic behavior of a droplet on a non-ideal surface subject to a shear flow. For the droplet pinned on the solid surface due to a large hysteresis window of contact angles, the predicted shapes of deformed droplet are in good agreement with the previous numerical results of Schleizer and Bonnecaze [74]. In the early stages (e.g. $\gamma t<6$ ), four typical motion modes of contact points, which were observed by Wang et al. [64], are qualitatively reproduced by adjusting the hysteresis windows. In addition, it is found that the viscosity ratio can affect not only the deformation and slipping velocity of the droplet but also its breakup and hysteresis behavior. To show the model's capability in dealing with complex boundaries, we simulate the droplet breakup process in a microfluidic T-junction, where one half of the channel surface is ideal and the other half is non-ideal. For all of the viscosity ratios, the droplet finally breaks up into two unequal-sized daughter droplets with the smaller one in the non-ideal branch. However, decreasing the viscosity ratio can strengthen the effect of contact angle hysteresis, thereby leading to an earlier breakup of the droplet and a decrease in the maximum length that the droplet can reach before the breakup. Also, the daughter droplet in the ideal branch always keeps moving towards the outlet, but the daughter droplet in the non-ideal branch either moves at an extremely low velocity or remains pinned at a position, which strongly depends on the value of viscosity ratio. In summary, our numerical results indicate that the present color-fluid LBM is numerically stable and accurate for simulating binary fluids with a wide range of viscosity ratios, no matter whether the contact angle hysteresis is considered, and also can be easily extended for handling complex geometry.

\section{Acknowledgments}

This work is financially supported by the Thousand Youth Talents Program for Distinguished Young Scholars and the National Natural Science Foundation of China (No. 51406148).

[1] J. H. Snoeijer and B. Andreotti, Annu. Rev. Fluid Mech. 45, 269 (2013).

[2] P. G. de Gennes, Rev. Mod. Phys. 57, 827 (1985).

[3] R. G. Cox, J. Fluid Mech. 168, 169 (1986).

[4] R. L. Hoffman, J. Coll. Interf. Sci. 50, 228 (1975).

[5] T.-S. Jiang, O. Soo-Gun, and J. C. Slattery, J. Coll. Interf. Sci. 69, 74 (1979).

[6] A. Jabbarzadeh and R. I. Tanner, Rheology Reviews (2006), chap. Molecular dynamics simulation and its application in nano-rheology, pp. 165-216.

[7] B. Shi and V. K. Dhir, THE JOURNAL OF CHEMICAL PHYSICS 130, 034705 (2009).

[8] E. Bertrand, T. D. Blake, and J. D. Coninck, J. Phys.: Condens. Matter 21, 464124 (2009).

[9] F.-C. Wang and Y.-P. Zhao, Colloid Polym. Sci. 291, 307 (2013).

[10] C. Hirt and B. Nichols, J. Comput. Phys. 39, 201 (1981).

[11] D. Gueyffier, J. Li, A. Nadim, R. Scardovelli, and S. Zaleski, J. Comput. Phys. 152, 423 (1999), ISSN $0021-9991$.

[12] M. Sussman, E. Fatemi, P. Smereka, and S. Osher, Comput. Fluids 27, 663 (1998).

[13] S. Osher and R. P. Fedkiw, Level sets methods and dynamic implicit surfaces (Springer, 2003).

[14] L. M. Hocking, J. Fluid Mech. 79, 209 (1977).

[15] H.-Y. Chen, D. Jasnow, and J. Viñals, Phys. Rev. Lett. 85, 1686 (2000).

[16] J. Zhang, Microfluid. Nanofluid. 10, 1 (2011).

[17] C. K. Aidun and J. R. Clausen, Annu. Rev. Fluid Mech. 42, 439 (2010), ISSN 0066-4189.

[18] S. Chen and G. D. Doolen, Annu. Rev. Fluid Mech. 30, 329 (1998).

[19] A. K. Gunstensen, D. H. Rothman, S. Zaleski, and G. Zanetti, Phys. Rev. A 43, 4320 (1991).

[20] I. Halliday, R. Law, C. M. Care, and A. Hollis, Phys. Rev. E 73, 056708 (pages 11) (2006).

[21] T. Reis and T. N. Phillips, J. Phys. A-Math. Theor. 40, 4033 (2007).

[22] H. Liu, A. J. Valocchi, and Q. Kang, Phys. Rev. E 85, 046309 (2012).

[23] M. R. Swift, E. Orlandini, W. R. Osborn, and J. M. Yeomans, Phys. Rev. E 54, 5041 (1996).

[24] H. Zheng, C. Shu, and Y. Chew, J. Comput. Phys. 218, 353 (2006).

[25] T. Lee and L. Liu, J. Comput. Phys. 229, 8045 (2010).

[26] X. Shan and H. Chen, Phys. Rev. E 47, 1815 (1993).

[27] X. Shan and H. Chen, Phys. Rev. E 49, 2941 (1994).

[28] M. Sbragaglia, R. Benzi, L. Biferale, S. Succi, K. Sugiyama, and F. Toschi, Phys. Rev. E 75, 026702 (2007).

[29] X. He, S. Chen, and R. Zhang, J. Comput. Phys. 152, 642 (1999).

[30] R. R. Nourgaliev, T. N. Dinh, T. G. Theofanous, and D. Joseph, International Journal of Multiphase Flow 29, 117 (2003), ISSN 0301-9322. 
[31] H. Liu and Y. Zhang, Phys. Fluids 23, 082101 (2011).

[32] A. Gupta and R. Kumar, Microfluidics and Nanofluidics 8, 799 (2010).

[33] H. Liu, A. J. Valocchi, C. Werth, Q. Kang, and M. Oostrom, Adv. Water Resour. 73, 144 (2014).

[34] Y. Ba, H. Liu, J. Sun, and R. Zheng, Phys. Rev. E 88 88, 043306 (2013).

[35] A. P. Hollis, T. J. Spencer, I. Halliday, and C. M. Care, IMA J. Appl. Math. 76, 726 (2011).

[36] J.-B. Dupont and D. Legendre, J. Comput. Phys. 229, 2453 (2010).

[37] C. M. Pooley, H. Kusumaatmaja, and J. M. Yeomans, Phys. Rev. E 78, 056709 (2008).

[38] M. L. Porter, E. T. Coon, Q. Kang, J. D. Moulton, and J. W. Carey, Phys. Rev. E 86, 036701 (2012).

[39] H. Liu, A. J. Valocchia, Y. Zhang, and Q. Kang, J. Comput. Phys. 256, 334 (2014).

[40] H. DING and P. D. M. SPELT, J. Fluid Mech. 599, 341 (2008).

[41] H. Ding and P. D. M. Spelt, Phys. Rev. E 75, 046708 (2007).

[42] S. V. Lishchuk, C. M. Care, and I. Halliday, Phys. Rev. E 67, 036701 (2003).

[43] I. Halliday, A. P. Hollis, and C. M. Care, Phys. Rev. E 76, 026708 (2007).

[44] H. Liu, Y. Zhang, and A. J. Valocchi, J. Comput. Phys. 231, 4433 (2012).

[45] P. Lallemand and L.-S. Luo, Phys. Rev. E 61, 6546 (2000).

[46] D. d'Humières, I. Ginzburg, M. Krafczyk, P. Lallemand, and L.-S. Luo, Philos. Trans. R. Soc. A 360, 437 (2002).

[47] Y. H. Qian, D. D'Humières, and P. Lallemand, Europhys. Lett. 17, 479 (1992).

[48] Z. Yu and L.-S. Fan, Phys. Rev. E 82, 046708 (2010).

[49] J. U. Brackbill, D. B. Kothe, and C. Zemach, J. Comput. Phys. 100, 335 (1992), ISSN 0021-9991.

[50] A. J. C. Ladd and R. Verberg, J. Stat. Phys. 104, 1191 (2001).

[51] Z. Guo, C. Zheng, and B. Shi, Phys. Rev. E 65, 046308 (2002).

[52] Z. Chai and T. S. Zhao, Phys. Rev. E 86, 016705 (2012).

[53] L.-S. Luo, W. Liao, X. Chen, Y. Peng, and W. Zhang, Phys. Rev. E 83, 056710 (2011).

[54] M. E. McCracken and J. Abraham, Phys. Rev. E 71, 036701 (2005).

[55] Y. Q. Zu and S. He, Phys. Rev. E 87, 043301 (2013).

[56] I. Ginzbourg and P. Adler, Transp. Porous Med. 20, 37 (1995).

[57] A. Coward, Y. Renardy, M. Renardy, and J. Richards, J. Comput. Phys. 132, 346 (1997).

[58] M. Latva-Kokko and D. H. Rothman, Phys. Rev. E 71, 056702 (2005).

[59] J.-J. Huang, H. Huang, and X. Wang, Phys. Fluids 26, 062101 (2014).

[60] A. J. C. Ladd, J. Fluid Mech. 271, 285 (1994).

[61] H. Liu and Y. Zhang, J. Comput. Phys. 280, 37 (2015).

[62] P. Dimitrakopoulos and J. Higdon, J. Fluid Mech. 395 (1999).

[63] P. D. Spelt, J. Comput. Phys. 207, 389 (2005).

[64] L. Wang, H. bo Huang, and X.-Y. Lu, Phys. Rev. E 87, 013301 (2013).

[65] R. Tadmor, Langmuir 20, 7659 (2004).

[66] N. Moradi, F. Varnik, and I. Steinbach, EPL (Europhysics Letters) 89, 26006 (2010).

[67] N. Moradi, F. Varnik, and I. Steinbach, EPL (Europhysics Letters) 95, 44003 (2011).

[68] F. Varnik, M. Gross, N. Moradi, G. Zikos, P. Uhlmann, P. Müller-Buschbaum, D. Magerl, D. Raabe, I. Steinbach, and M. Stamm, J. Phys.: Condens. Matter 23, 184112 (2011).

[69] H. Liu, Q. Kang, C. R. Leonardi, B. D. Jones, S. Schmieschek, A. Narvaez, J. R. Williams, A. J. Valocchi, and J. Harting, arXiv preprint arXiv:1404.7523 (2014).

[70] B. Dong, Y. Y. Yan, and W. Z. Li, Transp. Porous Med. 88, 293 (2011).

[71] E. W. Washburn, Phys. Rev. 17, 273 (1921).

[72] F. Diotallevi, L. Biferale, S. Chibbaro, A. Lamura, G. Pontrelli, M. Sbragaglia, S. Succi, and F. Toschi, Eur. Phys. J. Special Topics 166, 111 (2009).

[73] C. Pooley, H. Kusumaatmaja, and J. Yeomans, Eur. Phys. J. Special Topics 171, 63 (2009).

[74] A. D. Schleizer and R. T. Bonnecaze, J. Fluid Mech. 383, 29 (1999).

[75] Q. Zou and X. He, Phys. Fluids 9, 1591 (1997).

[76] M.-C. Jullien, M.-J. T. M. Ching, C. Cohen, L. Menetrier, and P. Tabeling, Phys. Fluids 21, 072001 (2009).

[77] A. M. Leshansky, S. Afkhami, M.-C. Jullien, and P. Tabeling, Phys. Rev. Lett. 108, 264502 (2012).

[78] X. Wang, C. Zhu, T. Fu, and Y. Ma, AIChE J. 61, 1081 (2015). 